\title{
MATHEMATICAL MODELLING AND NUMERICAL SOLUTION OF SWELLING OF CARTILAGINOUS TISSUES. PART II: MIXED-HYBRID FINITE ELEMENT SOLUTION*
}

\author{
Kamyar Malakpoor $^{1}$, Enrique F. KaAsschieter ${ }^{2}$ and Jacques M. Huyghe ${ }^{3}$
}

\begin{abstract}
The swelling and shrinkage of biological tissues are modelled by a four-component mixture theory [J.M. Huyghe and J.D. Janssen, Int. J. Engng. Sci. 35 (1997) 793-802; K. Malakpoor, E.F. Kaasschieter and J.M. Huyghe, Mathematical modelling and numerical solution of swelling of cartilaginous tissues. Part I: Modeling of incompressible charged porous media. ESAIM: M2AN 41 (2007) 661-678]. This theory results in a coupled system of nonlinear parabolic differential equations together with an algebraic constraint for electroneutrality. In this model, it is desirable to obtain accurate approximations of the fluid flow and ions flow. Such accurate approximations can be determined by the mixed finite element method. The solid displacement, fluid and ions flow and electro-chemical potentials are taken as degrees of freedom. In this article the lowest-order mixed method is discussed. This results into a first-order nonlinear algebraic equation with an indefinite coefficient matrix. The hybridization technique is then used to reduce the list of degrees of freedom and to speed up the numerical computation. The mixed hybrid finite element method is then validated for small deformations using the analytical solutions for one-dimensional confined consolidation and swelling. Two-dimensional results are shown in a swelling cylindrical hydrogel sample.
\end{abstract}

Mathematics Subject Classification. 35K55, 35M10, 65F10, 65M60.

Received July 12, 2006. Revised March 12, 2007.

\section{Notation}

$\begin{array}{ll}a & \text { scalar } \\ \mathbf{a}, \mathfrak{a} & \text { vector } \\ A & \text { scalar } \\ \mathbf{A}, \mathfrak{A} & \text { matrix } \\ \mathcal{A} & \text { tensor } \\ \nabla & \text { gradient operator in current configuration } \\ \nabla_{0} & \text { gradient operator in initial configuration }\end{array}$

\footnotetext{
Keywords and phrases. Hydrated soft tissue, nonlinear parabolic partial differential equation, mixed hybrid finite element.

* This work was supported by the Netherlands Organization for Science Research (NWO), through the project 613.002.050.

1 Korteweg-de Vries Institute for Mathematics (Faculty NWI), University of Amsterdam, Plantage Muidergracht 24, 1018 TV, Amsterdam, The Netherlands. k.malakpoor@uva.nl

2 Departement of Mathematics and Computer Science, Eindhoven University of Technology, PO Box 513 , 5600 MB Eindhoven, The Netherlands. e.f.kaasschieter@tue.nl

3 Faculty of Mechanical Engineering, Eindhoven University of Technology, PO Box 513, 5600 MB Eindhoven, The Netherlands. j.m.r.huyghe@tue.nl

(C) EDP Sciences, SMAI 2007
} 


\section{Nomenclature}

\begin{tabular}{|c|c|c|}
\hline $\mathcal{C}$ & right Cauchy-Green strain tensor & {$[-]$} \\
\hline$c$ & molar concentration of the fluid phase & {$\left[\mathrm{mol} \cdot \mathrm{m}^{-3}\right]$} \\
\hline$c^{\beta}$ & molar concentration of ion $\beta$ per unit fluid volume & {$\left[\mathrm{mol} \cdot \mathrm{m}^{-3}\right]$} \\
\hline$c^{f c}$ & molar concentration of fixed charge per unit fluid volume & {$\left[\mathrm{mol} \cdot \mathrm{m}^{-3}\right]$} \\
\hline $\mathcal{D}^{\beta}$ & diffusivity of ion $\beta$ & {$\left[\mathrm{m}^{2} \cdot \mathrm{s}^{-1}\right]$} \\
\hline $\mathcal{E}$ & strain tensor & {$[-]$} \\
\hline$f^{\beta}$ & activity coefficient of ion $\beta$ & {$[-]$} \\
\hline$F$ & Faraday's constant & {$\left[\mathrm{C} \cdot \mathrm{mol}^{-1}\right]$} \\
\hline $\mathcal{K}$ & hydraulic permeability & {$\left[\mathrm{m}^{4} \cdot \mathrm{N}^{-1} \cdot \mathrm{s}^{-1}\right]$} \\
\hline$p$ & pressure of the fluid phase & {$\left[\mathrm{N} \cdot \mathrm{m}^{-2}\right]$} \\
\hline $\mathbf{q}^{l}$ & specific discharge relative to the solid & {$\left[\mathrm{m} \cdot \mathrm{s}^{-1}\right]$} \\
\hline $\mathbf{q}^{\beta}$ & flux of ion $\beta$ relative to the fluid & {$\left[\mathrm{mol} \cdot \mathrm{m}^{-2} \cdot \mathrm{s}^{-1}\right]$} \\
\hline$R$ & universal gas constant & {$\left[\mathrm{J} \cdot \mathrm{mol}^{-1} \cdot \mathrm{K}^{-1}\right.$} \\
\hline $\mathcal{S}$ & second Piola-Kirchhoff stress & {$\left[\mathrm{N} \cdot \mathrm{m}^{-2}\right]$} \\
\hline$t$ & time & {$[\mathrm{s}]$} \\
\hline$T$ & absolute temperature & {$[\mathrm{K}]$} \\
\hline $\mathbf{u}$ & displacement & [m] \\
\hline $\bar{V}^{\beta}$ & partial molar volume of ion $\beta$ & {$\left[\mathrm{m}^{3} \cdot \mathrm{mol}^{-1}\right]$} \\
\hline $\mathbf{v}^{\alpha}$ & velocity of the $\alpha$-phase & {$\left[\mathrm{m} \cdot \mathrm{s}^{-1}\right]$} \\
\hline $\mathbf{v}^{\beta}$ & velocity of ion $\beta$ & {$\left[\mathrm{m} \cdot \mathrm{s}^{-1}\right]$} \\
\hline$z^{\beta}$ & valency of ion $\beta$ & 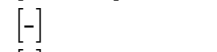 \\
\hline$z^{f c}$ & valency of fixed charge & [-] \\
\hline$\sigma$ & Cauchy stress tensor & {$\left[\mathrm{N} \cdot \mathrm{m}^{-2}\right]$} \\
\hline$\lambda_{s}$ & Lamé stress constant & {$\left[\mathrm{N} \cdot \mathrm{m}^{-2}\right]$} \\
\hline$\mu^{l}$ & electro-chemical potential of the fluid phase & {$\left[\mathrm{N} \cdot \mathrm{m}^{-2}\right]$} \\
\hline$\mu^{\beta}$ & electro-chemical potential of ion $\beta$ & {$\left[\mathrm{J} \cdot \mathrm{mol}^{-1}\right]$} \\
\hline$\mu_{s}$ & Lamé stress constant & {$\left[\mathrm{N} \cdot \mathrm{m}^{-2}\right]$} \\
\hline$\Pi$ & first Piola-Kirchhoff stress & {$\left[\mathrm{N} \cdot \mathrm{m}^{-2}\right]$} \\
\hline$\xi$ & voltage & [V] \\
\hline$\rho^{\alpha}$ & bulk density of the $\alpha$-phase & {$\left[\mathrm{kg} \cdot \mathrm{m}^{-3}\right]$} \\
\hline$\rho_{T}^{\alpha}$ & true density of the $\alpha$-phase & {$\left[\mathrm{kg} \cdot \mathrm{m}^{-3}\right]$} \\
\hline$\varphi$ & volume fraction of the liquid phase & \\
\hline$\varphi^{\alpha}$ & volume fraction of the $\alpha$-phase & \\
\hline$\varphi^{\beta}$ & volume fraction of the component $\beta$ & [ \\
\hline$\Gamma^{\beta}$ & osmotic coefficient of ion $\beta$ & \\
\hline
\end{tabular}

\section{INTRODUCTION}

Cartilaginous tissues are soft hydrated tissues with strong swelling and shrinkage properties. This swelling and shrinkage behaviour of cartilaginous tissues is caused by the flow of water that is bound to the charged, solid skeleton of porous tissue. The driving mechanism is an interplay of mechanical, chemical and electrical forces. In Part I [11], a finite deformation four component model has been derived to account for osmotic effects. To account for finite deformation the set of equations is written in Lagrangian coordinates. This leads to a 
system of couple time-dependent non-linear equations together with boundary conditions as follows:

$$
\begin{array}{|rl}
\hline \text { Balance equations } & \\
\hline \nabla_{0} \cdot\left(\mathcal{S F}^{T}\right) & =\mathbf{0}, \\
\frac{D J \varphi}{D t}+\nabla_{0} \cdot \mathfrak{G}^{l} & =0, \\
\frac{D J \varphi c^{\beta}}{D t}+\nabla_{0} \cdot\left(\mathfrak{d}^{\beta}+c^{\beta} \mathfrak{U}^{l}\right) & =0, \quad \beta=+,-. \\
\hline \text { Constitutive equations } & \\
\hline \frac{\partial W}{\partial \mathcal{E}}-p J \mathcal{C}^{-1} & =\mathcal{S}, \\
-\widetilde{\mathcal{K}}\left(\nabla_{0} \mu^{l}+c^{+} \nabla_{0} \mu^{+}+c^{-} \nabla_{0} \mu^{-}\right) & =\mathfrak{d}^{l}, \\
-\frac{\widetilde{\mathcal{D}}^{\beta} c^{\beta} \varphi}{R T} \nabla_{0} \mu^{\beta} & =\mathfrak{G}^{\beta}, \quad \beta=+,-.
\end{array}
$$

where $\mathcal{F}, J, \mathcal{C}, \nabla_{0}, \frac{D}{D t}$ and $W$ are the deformation gradient of the solid phase, its determinant, the right Cauchy-Green strain tensor, the Gradient operator in initial configuration, the material time derivative and the Helmholtz free energy, respectively. Also

$$
\begin{aligned}
\mathfrak{Q}^{\beta} & =J \mathcal{F}^{-1} \mathbf{q}^{\beta}, \quad \beta=l,+,-, \\
\widetilde{\mathcal{K}} & =J \mathcal{F}^{-1} \mathcal{K} \mathcal{F}^{-T}, \\
\widetilde{\mathcal{D}}^{\beta} & =J \mathcal{F}^{-1} \mathcal{D}^{\beta} \mathcal{F}^{-T}, \quad \beta=+,-.
\end{aligned}
$$

In this paper we keep all the assumptions from [11], but we also assume infinitesimal deformation for the solid phase. In the infinitesimal theory of elasticity it is assumed that the components of the displacement vector and their spatial derivatives are infinitesimal of the first order so that we neglect products and squares of these quantities in comparison with their first powers [4], page 6. Using this approximation we find the deformation tensor and the strain tensor as

$$
\mathcal{F}=\mathcal{I}+\nabla_{0} \mathbf{U}, \quad \mathcal{E}=\frac{1}{2}\left(\nabla_{0} \mathbf{U}+\left(\nabla_{0} \mathbf{U}\right)^{T}\right)
$$

where $\mathbf{U}=\mathbf{x}-\mathbf{X}$ is the displacement vector.

Recall [11], equation (4.1), that

$$
\varphi=1-\varphi^{s}=1-\frac{1-\varphi_{0}}{J} .
$$

Since the solid phase is assumed to have infinitesimal deformation, the Taylor linearisation for $J^{-1}$ at $\mathcal{F}=\mathbf{I}$ implies

In the above relation, we use

$$
J^{-1} \approx 1-\left.\left(\frac{1}{J^{2}} \frac{\partial J}{\partial \mathcal{F}}\right)\right|_{\mathcal{F}=\mathcal{I}}:(\mathcal{F}-\mathcal{I})=1-\nabla_{0} \cdot \mathbf{U}
$$

$$
\frac{\partial J}{\partial \mathcal{F}}=J \mathcal{F}^{-T}
$$

Putting the linearized form of $J^{-1}$ into (1.3) results into

$$
\varphi=1-\left(1-\varphi_{0}\right)\left(1-\nabla_{0} \cdot \mathbf{U}\right) .
$$


Also remember the relation for fixed charges density in [11], equation (4.7), given by

$$
c^{f c}=c_{0}^{f c} \varphi_{0}\left(J-\varphi_{0}^{s}\right)^{-1} .
$$

From the assumption of infinitesimal elastic deformation for the solid phase, the Taylor linearisation for the function $\left(J-\varphi_{0}^{s}\right)^{-1}$ at $\mathcal{F}=\mathcal{I}$ results into

$$
\left(J-\varphi_{0}^{s}\right)^{-1} \approx\left(1-\varphi_{0}^{s}\right)^{-1}-\left.\left(\frac{1}{\left(J-\varphi_{0}^{s}\right)^{2}} \frac{\partial J}{\partial \mathcal{F}}\right)\right|_{\mathcal{F}=\mathbf{I}}:(\mathcal{F}-\mathcal{I}) .
$$

Hence

$$
c^{f c}=c_{0}^{f c}\left(1-\frac{\nabla_{0} \cdot \mathbf{U}}{\varphi_{0}}\right) .
$$

We choose a linear elastic material and therefore the elastic energy part is of the form:

$$
W(\mathcal{E})=\mu_{s} \mathcal{E}: \mathcal{E}+\frac{\lambda_{s}}{2}\left(\nabla_{0} \cdot \mathbf{U}\right)^{2},
$$

where $\lambda_{s}$ and $\mu_{s}$ are the Lamé stress constants.

In the next step we will rewrite the equations in the infinitesimal deformation regime. Starting from the force balance and the related constitutive equation we have

$$
\begin{aligned}
& \nabla_{0} \cdot\left(\frac{\partial W}{\partial \mathcal{E}} \mathcal{F}^{T}\right)-\nabla_{0} \cdot\left(p J \mathcal{F}^{-1} \mathcal{I}\right)=\mathbf{0}, \\
\text { or } \quad & \nabla_{0} \cdot\left(\frac{\partial W}{\partial \mathcal{E}} \mathcal{F}^{T}\right)-J \nabla p=\mathbf{0}, \\
\text { or } \quad & \nabla_{0} \cdot\left(\frac{\partial W}{\partial \mathcal{E}} \mathcal{F}^{T}\right)-J \mathcal{F}^{-T} \nabla_{0} p=\mathbf{0} .
\end{aligned}
$$

By using (1.2) the last equation is reduced to

$$
\begin{aligned}
& \nabla_{0} \cdot\left(2 \mu_{s} \mathcal{E}+\lambda_{s} \nabla_{0} \mathbf{U}\right)-\nabla_{0} p+\nabla_{0} \cdot\left(\left(2 \mu_{s} \mathcal{E}+\lambda_{s} \nabla_{0} \mathbf{U}\right) \nabla_{0} \mathbf{U}^{T}\right) \\
+ & \nabla_{0} \mathbf{U}^{T} \nabla_{0} p-\nabla_{0} \cdot \mathbf{U} \nabla_{0} p+\nabla_{0} \cdot \mathbf{U} \nabla_{0} \mathbf{U}^{T} \nabla_{0} p=\mathbf{0} .
\end{aligned}
$$

Assuming infinitesimal deformation for the solid phase, all terms except first and second terms are vanished and finally we have

$$
\nabla_{0} \cdot\left(2 \mu_{s} \mathcal{E}+\lambda_{s} \nabla_{0} \mathbf{U}\right)-\nabla_{0} p=\mathbf{0} .
$$

Note that from (1.5), we have

$$
J \varphi=(1+\nabla \cdot \mathbf{U})\left(1-\left(1-\varphi_{0}\right)(1-\nabla \cdot \mathbf{U})\right)=\nabla \cdot \mathbf{U}+\varphi_{0} .
$$

The last assumption in this paper is that, the permeability and diffusion tensors are considered to be isotropic tensors represented by scalar multiple of the identity, thus in the Lagrangian coordinates $\mathcal{D}^{\beta}$ and $\mathcal{K}$ are constants. To make the notations simpler, we change notations according to the following table:

\begin{tabular}{|l|c|c|c|c|c|c|}
\hline Old notations & $\mathbf{U}$ & $\nabla_{0}$ & $\frac{D}{D t}$ & $\mathfrak{a}^{\beta}, \beta=l,+,-$ & $\widetilde{\mathcal{D}}^{\beta}, \beta=+,-$ & $\widetilde{\mathcal{K}}$ \\
\hline New notations & $\mathbf{u}$ & $\nabla$ & $\frac{\partial}{\partial t}$ & $\mathbf{q}^{\beta}, \beta=l,+,-$ & $D^{\beta}, \beta=+,-$ & $K$ \\
\hline
\end{tabular}


Let $\Omega$ be an open domain in $\mathbb{R}^{n}, n=1,2,3$, then define $\Omega_{T}=\Omega \times(0, T]$ for $T>0$, and consider the sets $\Gamma_{\mathbf{u}}^{D}$ and $\Gamma_{\mathbf{u}}^{N}$ (and similarly $\Gamma_{p}^{D}$ and $\Gamma_{p}^{N}$ ) to be two disjoint open subsets of the total boundary $\Gamma=\partial \Omega$, such that $\Gamma_{\alpha}^{D} \cap \Gamma_{\alpha}^{N}=\varnothing$ and $\bar{\Gamma}_{\alpha}^{D} \cup \bar{\Gamma}_{\alpha}^{N}=\Gamma$ for $\alpha=\mathbf{u}$ and $p$. We assume meas $\Gamma_{\alpha}^{D}>0$ for $\alpha=\mathbf{u}, p$. Now the total set of equations are given as:

\begin{tabular}{|c|c|c|}
\hline \multicolumn{3}{|r|}{ Conservation equations } \\
\hline $\begin{array}{l}\mathbf{0} \\
0\end{array}$ & $\begin{array}{l}= \\
= \\
=\end{array}$ & $\begin{array}{l}\nabla \cdot \boldsymbol{\sigma}-\nabla p \\
\frac{\partial \nabla \cdot \mathbf{u}}{\partial t}+\nabla \cdot \mathbf{q}^{l}, \\
\frac{\partial\left(\nabla \cdot \mathbf{u}+\varphi_{0}\right) c^{\beta}}{\partial t}+\nabla \cdot\left(\mathbf{q}^{\beta}+c^{\beta} \mathbf{q}^{l}\right), \quad \beta=+,-.\end{array}$ \\
\hline \multicolumn{3}{|r|}{ Constitutive equations } \\
\hline $\begin{array}{c}\sigma \\
\mathbf{q}^{l} \\
\mathbf{q}^{\beta}\end{array}$ & $\begin{array}{l}= \\
=\end{array}$ & $\begin{array}{l}2 \mu_{s} \mathcal{E}+\lambda_{s} \operatorname{tr} \mathcal{E}, \quad \mathcal{E}=\frac{1}{2}\left(\nabla \mathbf{u}+\left(\nabla \mathbf{u}^{T}\right)\right) \\
-K\left(\nabla \mu^{l}+c^{+} \nabla \mu^{+}+c^{-} \nabla \mu^{-}\right) \\
-\frac{D^{\beta}}{R T} \varphi c^{\beta} \nabla \mu^{\beta}, \quad \beta=+,-\end{array}$ \\
\hline \multicolumn{3}{|r|}{ Secondary equations } \\
\hline$\varphi$ & $=$ & $1-\left(1-\varphi_{0}\right)(1-\nabla \cdot \mathbf{u}), \quad c^{f c}=c_{0}^{f c}\left(1-\frac{\nabla \cdot \mathbf{u}}{\varphi_{0}}\right)$ \\
\hline$c^{\beta}$ & $=$ & 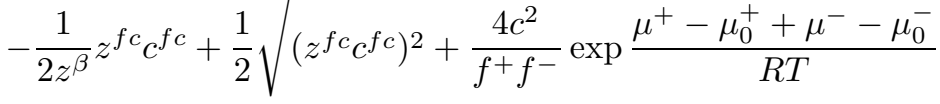 \\
\hline $\begin{array}{l}p \\
\xi\end{array}$ & $\begin{array}{l}= \\
=\end{array}$ & $\begin{array}{l}\mu^{l}-\mu_{0}^{l}+R T\left(\Gamma^{+} c^{+}+\Gamma^{-} c^{-}\right), \\
\frac{1}{z^{\beta} F}\left(\mu^{\beta}-\mu_{0}^{\beta}-R T \ln \frac{f^{\beta} c^{\beta}}{c}\right), \quad \beta=+,-.\end{array}$ \\
\hline \multicolumn{3}{|r|}{ Boundary conditions } \\
\hline $\begin{array}{r}\mathbf{u} \\
\mu^{\beta} \\
\mathbf{g}_{\mathbf{u}}^{N} \\
\mathbf{n} \cdot \mathbf{q}^{\beta}\end{array}$ & $\begin{array}{l}= \\
= \\
= \\
=\end{array}$ & $\begin{array}{ll}\mathbf{0} & \text { on } \Gamma_{\mathbf{u}}^{D} \times(0, T], \\
\mu_{i n}^{\beta} & \text { on } \Gamma_{p}^{D} \times(0, T], \quad \beta=l,+,- \\
\mathbf{n} \cdot(\boldsymbol{\sigma}(\mathbf{u})-p \boldsymbol{I}) & \text { on } \Gamma_{\mathbf{u}}^{N} \times(0, T], \\
0 & \text { on } \Gamma_{p}^{N} \times(0, T], \quad \beta=l,+,-.\end{array}$ \\
\hline
\end{tabular}

In the above equations $\mu_{0}^{l}$ and $\mu_{0}^{\beta}, \beta=+,-$ are the initial electro-chemical potential of the fluid phase and ion $\beta$, respectively; $\Gamma^{\beta} \in(0,1], \beta=+,-$ is the osmotic coefficient, the activity $f^{\beta}$ is defined by

$$
f^{\beta}=\left(\frac{c^{\beta}}{c}\right)^{\Gamma^{\beta}-1}, \quad \beta=+,-.
$$

We assume that the electro-chemical potentials are continuous at the boundary, i.e.,

$$
\mu_{i n}^{\beta}=\mu_{o u t}^{\beta}, \quad \beta=l,+,-,
$$


where subscript 'in' and 'out' refer to the electro-chemical potentials in the outer and inner solutions, respectively. Assume that $\Gamma_{\text {in }}^{+}=\Gamma_{\text {in }}^{-}=\Gamma$ and $\Gamma_{\text {out }}^{+}=\Gamma_{\text {out }}^{-}=1$, then

$$
\begin{aligned}
& \mu_{\text {in }}^{l}=\mu_{0}^{l}+p_{\text {out }}-2 R T c_{\text {out }}, \\
& \mu_{\text {in }}^{\beta}=\mu_{0}^{\beta}+F z^{\beta} \xi_{\text {out }}+R T \Gamma \ln \frac{c_{\text {out }}}{c},
\end{aligned}
$$

where, $c_{\text {out }}, p_{\text {out }}$ and $\xi_{\text {out }}$ are the ions concentration, fluid pressure and the electrical potential of the outer solution, respectively.

In our model it is desirable to obtain approximations of the fluid flow and ions flow that fulfill the conservation equations. In finite element simulations [17], these quantities are generally calculated by differentiation of the electro-chemical potentials solution. This approach may lead to violation of the mass conservation principle. The mixed finite element method provides an attractive framework for this type of problems by simultaneously approximating flows and electro-chemical potentials. Flows computed by mixed finite elements automatically satisfy the "divergence free" property, both locally and globally, and the corresponding normal flux field is guaranteed to be continuous across inter-element boundaries. The mixed finite element method has been extensively used for the solution of parabolic equations arising in different application fields. The mixed finite element method was proposed for two-dimensional problems by [14-16], and by Nédélec for three-dimensional problems $[12,13]$. In this article the mixed finite element variational formulation is derived for the set of coupled equations in general dimension. Only the lowest-order mixed method will be considered, first, because higher order-methods result in some conceptual complications and, second, because the lowest-order method is comparatively easy and straightforward to use for practical problems.

In steady-state flow problems, i.e. elliptic equations, the discretised system derived from mixed formulation becomes indefinite. A common solution method is so-called mixed-hybrid finite element (MHFEM) technique [5]. Through the definition of an extra variable representing the pressure at element edges, MHFEM gives rise to a symmetric positive definite matrix with good conditioning properties. Using the MHFEM technique for our model, we still have an indefinite system but the advantage is that the number of degrees of freedom will be reduced. In fact, for a three-dimensional problem this number will be reduced from 15 to 6 degrees of freedom. Note that in our case the equations are time dependent and non-linear, therefore a choice of time integration and nonlinear solver is needed. We use some techniques to tackle this problem. In [10] the analytical solution are given for a linearized problem. Finally the results are validated for confined consolidation and free swelling experiments using the analytical solution.

This paper is outlined as follows: Section 2 considers the coupled mixed formulation of the problem with some preliminary definitions of function spaces. In this section we introduce the MHFEM technique and following the theory we derived a nonlinear algebraic equation for the displacement as unknown. Section 3 is devoted to the numerical simulations of one-dimensional confined consolidation and swelling experiments. Furthermore, in this section we consider a two dimensional swelling experiment.

\section{The COUPLED MIXED FORMULATION}

\subsection{Notations and preliminaries}

In this section we will introduce some notations and definitions crucial for the mixed formulation [3].

Throughout this article, $\Omega$ shall denote a bounded, open, connected subset of $\mathbb{R}^{n}, n=1,2,3$, with a Lipschitz continuous boundary $\Gamma$ (see [3], p. 12). $L^{2}(\Omega)$ is the set of all measurable scalar functions $f: \Omega \rightarrow \mathbb{R}$ such that

$$
\|f\|_{0}=\left(\int_{\Omega} f^{2} \mathrm{~d} \mathbf{x}\right)^{1 / 2}<\infty .
$$


$\mathbf{L}^{2}(\Omega)$ is the set of all measurable vector functions $\mathbf{f}: \Omega \rightarrow \mathbb{R}^{n}$ such that

$$
\|\mathbf{f}\|_{0}=\left(\int_{\Omega}|\mathbf{f}|^{2} \mathrm{~d} \mathbf{x}\right)^{1 / 2}<\infty .
$$

Let $k$ be a nonnegative integer, then $H^{k}(\Omega)$ denotes the Sobolev space,

$$
H^{k}(\Omega)=\left\{v \in L^{2}(\Omega): D^{\alpha} v \in L^{2}(\Omega) \text { for all }|\alpha| \leq k\right\},
$$

equipped with the norm

$$
\|v\|_{k}=\sum_{|\alpha| \leq k}\left\|D^{\alpha} v\right\|_{0} .
$$

Let $C_{0}^{\infty}(\Omega)$ denote the space of all infinitely differentiable scalar functions $\varphi: \Omega \rightarrow \mathbb{R}$ with compact support in $\Omega$. We denote by $H_{0}^{k}(\Omega)$ the closure of the space $C_{0}^{\infty}(\Omega)$ in $H^{k}(\Omega)$. Moreover if we define

$$
|v|_{k}=\sum_{|\alpha|=k}\left\|D^{\alpha} v\right\|_{0}
$$

then $|\cdot|_{k}$ and $\|\cdot\|_{k}$ are equivalent norms in $H_{0}^{k}(\Omega)$. We denote by $H^{-k}(\Omega)$ the dual space to $H_{0}^{k}(\Omega)$. For $f \in H^{-k}(\Omega)$,

$$
\|f\|_{-k}=\sup _{0 \neq v \in H_{0}^{k}(\Omega)} \frac{\langle f, v\rangle}{\|v\|_{k}}
$$

defines the related norm, where $\langle\cdot, \cdot\rangle$ denotes the duality pairing between $H^{-k}(\Omega)$ and $H_{0}^{k}(\Omega)$.

Let $k=1$, for $\varphi \in H^{1}(\Omega)$, the trace $\gamma_{D} \varphi=\left.\varphi\right|_{\Gamma}$ is well defined and is in $L^{2}(\Gamma)$. In other words there exists a constant $C$, depending only on $\Omega$, such that

$$
\|\varphi\|_{0, \Gamma} \leq C\left\|_{\varphi}\right\|_{1} \text { for all } \varphi \in H^{1}(\Omega)
$$

The image of the above trace mapping is denoted by

$$
H^{1 / 2}(\Gamma)=\left\{\gamma_{D} \varphi: \varphi \in H^{1}(\Omega)\right\}
$$

and is a Hilbert space with norm

$$
\|\psi\|_{1 / 2}=\inf _{\varphi \in H^{1}(\Omega)}\left\{\|\varphi\|_{1}: \psi=\gamma_{D} \varphi\right\}
$$

The space $H(\operatorname{div} ; \Omega)$ is defined by

$$
H(\operatorname{div} ; \Omega)=\left\{\mathbf{q} \in \mathbf{L}^{2}(\Omega): \nabla \cdot \mathbf{q} \in L^{2}(\Omega)\right\} .
$$

$H(\operatorname{div} ; \Omega)$ is a Hilbert space with respect to the norm

$$
\|\mathbf{q}\|_{\operatorname{div}}=\left\{\|\mathbf{q}\|_{0}^{2}+\|\nabla \cdot \mathbf{q}\|_{0}^{2}\right\}^{1 / 2} .
$$

We shall use the following version of Green's formula:

$$
\int_{\Omega}(\varphi \nabla \cdot \mathbf{q}+\nabla \varphi \cdot \mathbf{q}) \mathrm{d} \mathbf{x}=\int_{\Gamma} \varphi \mathbf{q} \cdot \mathbf{n} \mathrm{d} s
$$

for all $\mathbf{q} \in H(\operatorname{div} ; \Omega)$ and $\varphi \in H^{1}(\Omega)$. 
We also define the following linear spaces:

$$
\begin{aligned}
\mathcal{V} & =\left\{\mathbf{u} \in\left(H^{1}(\Omega)\right)^{n}: \mathbf{u}=0 \text { on } \Gamma_{\mathbf{u}}^{D}\right\}, \\
H_{D}^{1}(\Omega) & =\left\{\varphi \in H^{1}(\Omega): \varphi=0 \text { on } \Gamma_{p}^{D}\right\}, \\
H_{D}^{1 / 2}(\Gamma) & =\left\{\lambda \in H^{1 / 2}(\Gamma): \lambda=0 \text { on } \Gamma_{p}^{D}\right\}, \\
H_{N}(\operatorname{div} ; \Omega) & =\left\{\mathbf{q} \in H(\operatorname{div} ; \Omega): \mathbf{n} \cdot \mathbf{q}=0 \text { on } \Gamma_{p}^{N}\right\}, \\
H_{N}^{-1 / 2}(\Gamma) & =\left\{\mu \in H^{-1 / 2}(\Gamma): \mu=0 \text { on } \Gamma_{p}^{N}\right\} .
\end{aligned}
$$

\subsection{Mixed variational formulation of the problem}

For the preliminary step of defining a spatially semi-discrete approximate solution to our initial boundary value problem, we write the problem in weak form. We introduce a mixed variational formulation of the problem with the related boundary conditions. Define $\mathbf{q}_{\mathrm{tot}}^{\beta}=\mathbf{q}^{\beta}+c^{\beta} \mathbf{q}^{l}, \beta=+,-$. Then the mixed variational formulation of problem reads:

$$
\left\{\begin{array}{l}
\int_{\Omega}\left(2 \mu_{s} \mathcal{E}(\mathbf{u}): \mathcal{E}(\overline{\mathbf{u}})+\lambda_{s} \nabla \cdot \mathbf{u} \nabla \cdot \overline{\mathbf{u}}\right) \mathrm{d} \mathbf{x}-\int_{\Omega} \mu^{l} \nabla \cdot \overline{\mathbf{u}} \mathrm{d} \mathbf{x} \\
=\int_{\Omega}\left(R T\left(\Gamma^{+} c^{+}+\Gamma^{-} c^{-}\right)-\mu_{0}^{l}-p_{i n}\right) \nabla \cdot \overline{\mathbf{u}} \mathrm{d} \mathbf{x}+\int_{\Gamma_{\mathbf{u}}^{N}} \mathbf{g}_{\mathbf{u}}^{N} \cdot \overline{\mathbf{u}} \mathrm{d} s \\
\frac{1}{K} \int_{\Omega} \mathbf{q}^{l} \cdot \overline{\mathbf{q}}^{l} \mathrm{~d} \mathbf{x}-\int_{\Omega} \mu^{l} \nabla \cdot \overline{\mathbf{q}}^{l} \mathrm{~d} \mathbf{x}+\sum_{\beta=+,-} \int_{\Omega} c^{\beta} \nabla \mu^{\beta} \overline{\mathbf{q}}^{l} \mathrm{~d} \mathbf{x}=-\int_{\Gamma_{p}^{D}} \mu_{i n}^{l} \mathbf{n} \cdot \overline{\mathbf{q}}^{l} \mathrm{~d} s \\
\frac{R T}{D^{\beta}} \int_{\Omega} \mathbf{q}^{\beta} \cdot \overline{\mathbf{q}}^{\beta} \mathrm{d} \mathbf{x}+\int_{\Omega} \nabla \mu^{\beta} \overline{\mathbf{q}}^{\beta} \mathrm{d} \mathbf{x}=0, \beta=+,-, \\
-\int_{\Omega} \nabla \cdot \mathbf{q}^{l} \bar{\mu}^{l} \mathrm{~d} \mathbf{x}-\int_{\Omega} \frac{\partial \nabla \cdot \mathbf{u}}{\partial t} \bar{\mu}^{l} \mathrm{~d} \mathbf{x}=0, \\
-\int_{\Omega} \nabla \cdot \mathbf{q}_{\mathrm{tot}}^{\beta} \bar{\mu}^{\beta} \mathrm{d} \mathbf{x}=\int_{\Omega} \frac{\partial\left(\nabla \cdot \mathbf{u}+\varphi_{0}\right) c^{\beta}}{\partial t} \bar{\mu}^{\beta} \mathrm{d} \mathbf{x}, \beta=+,-,
\end{array}\right.
$$

where $\overline{\mathbf{q}}^{\beta}=\overline{\mathbf{q}}_{\mathrm{tot}}^{\beta}-c^{\beta} \overline{\mathbf{q}}^{l}$.

Note that the solution is time dependent. After summing up equations (2.1b) and (2.1d), using the fact that $\overline{\mathbf{q}}_{\text {tot }}^{\beta}$ is in $H_{N}(\operatorname{div} ; \Omega)$ and applying the Green's formula the above problem is rewritten as:

Find $\left(\mathbf{u}, \mathbf{q}^{l}, \mathbf{q}_{\text {tot }}^{+}, \mathbf{q}_{\text {tot }}^{-}, \mu^{l}, \mu^{+}, \mu^{-}\right)(\cdot, t) \in \mathcal{V} \times H_{N}(\operatorname{div} ; \Omega) \times H_{N}(\operatorname{div} ; \Omega) \times H_{N}(\operatorname{div} ; \Omega) \times L^{2}(\Omega) \times L^{2}(\Omega) \times L^{2}(\Omega)$, 
such that

$$
\left\{\begin{array}{l}
\int_{\Omega}\left(2 \mu_{s} \mathcal{E}(\mathbf{u}): \mathcal{E}(\overline{\mathbf{u}})+\lambda_{s} \nabla \cdot \mathbf{u} \nabla \cdot \overline{\mathbf{u}}\right) \mathrm{d} \mathbf{x}-\int_{\Omega} \mu^{l} \nabla \cdot \overline{\mathbf{u}} \mathrm{d} \mathbf{x} \\
=\int_{\Omega}\left(R T\left(\Gamma^{+} c^{+}+\Gamma^{-} c^{-}\right)-\mu_{0}^{l}-p_{i n}\right) \nabla \cdot \overline{\mathbf{u}} \mathrm{d} \mathbf{x}+\int_{\Gamma_{\mathbf{u}}^{N}} \mathbf{g}_{\mathbf{u}}^{N} \cdot \overline{\mathbf{u}} \mathrm{d} s \\
\frac{1}{K} \int_{\Omega} \mathbf{q}^{l} \cdot \overline{\mathbf{q}}^{l} \mathrm{~d} \mathbf{x}+\sum_{\beta=+,-} \frac{R T}{D^{\beta}} \int_{\Omega} \frac{\left(\mathbf{q}_{\mathrm{tot}}^{\beta}-c^{\beta} \mathbf{q}^{l}\right) \cdot\left(\overline{\mathbf{q}}_{\mathrm{tot}}^{\beta}-c^{\beta} \overline{\mathbf{q}}^{l}\right)}{\varphi c^{\beta}} \mathrm{d} \mathbf{x} \\
\quad-\int_{\Omega} \mu^{l} \nabla \cdot \overline{\mathbf{q}}^{l} \mathrm{~d} \mathbf{x}-\sum_{\beta=+,-} \int_{\Omega} \mu^{\beta} \nabla \cdot \overline{\mathbf{q}}_{\mathrm{tot}}^{\beta} \mathrm{d} \mathbf{x} \\
=-\int_{\Gamma_{p}^{D}} \mu_{i n}^{l} \mathbf{n} \cdot \overline{\mathbf{q}}^{l} \mathrm{~d} s-\sum_{\beta=+,-} \int_{\Gamma_{p}^{D}} \mu_{i n}^{\beta} \mathbf{n} \cdot \overline{\mathbf{q}}_{\mathrm{tot}}^{\beta} \mathrm{d} s \\
-\int_{\Omega} \nabla \cdot \mathbf{q}^{l} \bar{\mu}^{l} \mathrm{~d} \mathbf{x}-\int_{\Omega} \frac{\partial \nabla \cdot \mathbf{u}}{\partial t} \bar{\mu}^{l} \mathrm{~d} \mathbf{x}=0, \\
-\int_{\Omega} \nabla \cdot \mathbf{q}_{\mathrm{tot}}^{\beta} \bar{\mu}^{\beta} \mathrm{d} \mathbf{x}=\int_{\Omega} \frac{\partial\left(\nabla \cdot \mathbf{u}+\varphi_{0}\right) c^{\beta}}{\partial t} \bar{\mu}^{\beta} \mathrm{d} \mathbf{x}, \beta=+,-,
\end{array}\right.
$$

for all test functions $\left(\overline{\mathbf{u}}, \overline{\mathbf{q}}^{l}, \overline{\mathbf{q}}_{\text {tot }}^{+}, \overline{\mathbf{q}}_{\text {tot }}^{-}, \bar{\mu}^{l}, \bar{\mu}^{+}, \bar{\mu}^{-}\right) \in \mathcal{V} \times H_{N}(\operatorname{div} ; \Omega) \times H_{N}(\operatorname{div} ; \Omega) \times H_{N}(\operatorname{div} ; \Omega) \times L^{2}(\Omega) \times L^{2}(\Omega) \times$ $L^{2}(\Omega)$ and $t>0$.

Denote the triple $\left(\mathbf{q}^{l}, \mathbf{q}_{\text {tot }}^{+}, \mathbf{q}_{\text {tot }}^{-}\right)$and $\left(\mu^{l}, \mu^{+}, \mu^{-}\right)$by $\mathbf{q}$ and $\boldsymbol{\mu}$, respectively, and define:

$$
\begin{aligned}
a(\mathbf{u}, \overline{\mathbf{u}}) & =\int_{\Omega}\left(2 \mu_{s} \mathcal{E}(\mathbf{u}): \mathcal{E}(\overline{\mathbf{u}})+\lambda_{s} \nabla \cdot \mathbf{u} \nabla \cdot \overline{\mathbf{u}}\right) \mathrm{d} \mathbf{x} \\
b\left(\mathbf{u}, \bar{\mu}^{l}\right) & =-\int_{\Omega} \nabla \cdot \mathbf{u} \bar{\mu}^{l} \mathrm{~d} \mathbf{x}, \\
c(\mathbf{q}, \overline{\mathbf{q}}) & =\frac{1}{K} \int_{\Omega} \mathbf{q}^{l} \cdot \overline{\mathbf{q}}^{l} \mathrm{~d} \mathbf{x}+\sum_{\beta=+,-} \frac{R T}{D^{\beta}} \int_{\Omega} \frac{\left(\mathbf{q}_{\mathrm{tot}}^{\beta}-c^{\beta} \mathbf{q}^{l}\right) \cdot\left(\overline{\mathbf{q}}_{\mathrm{tot}}^{\beta}-c^{\beta} \overline{\mathbf{q}}^{l}\right)}{\varphi c^{\beta}} \mathrm{d} \mathbf{x}, \\
d^{l}\left(\mu^{l}, \overline{\mathbf{q}}^{l}\right) & =-\int_{\Omega} \mu^{l} \nabla \cdot \overline{\mathbf{q}}^{l} \mathrm{~d} \mathbf{x}, \\
d^{\beta}\left(\mu^{\beta}, \overline{\mathbf{q}}_{\mathrm{tot}}^{\beta}\right) & =-\int_{\Omega} \mu^{\beta} \nabla \cdot \overline{\mathbf{q}}_{\mathrm{tot}}^{\beta} \mathrm{d} \mathbf{x}, \quad \beta=+,-, \\
F_{(\overline{\mathbf{u}})} & =\int_{\Omega}\left(R T\left(\Gamma^{+} c^{+}+\Gamma^{-} c^{-}\right)-\mu_{0}^{l}-p_{\text {in }}\right) \nabla \cdot \overline{\mathbf{u}} \mathrm{d} \mathbf{x}+\int_{\Gamma_{\mathbf{u}}^{N}} \mathbf{g}_{\mathbf{u}}^{N} \cdot \overline{\mathbf{u}} \mathrm{d} s \\
F_{1}(\overline{\mathbf{q}}) & =-\int_{\Gamma} \mu_{i n}^{l} \mathbf{n} \cdot \overline{\mathbf{q}}^{l}-\sum_{\beta=+,-} \mu_{i n}^{\beta} \mathbf{n} \cdot \overline{\mathbf{q}}_{\mathrm{tot}}^{\beta} \mathrm{d} s, \\
F_{2}^{\beta}\left(\bar{\mu}^{\beta}\right) & =\int_{\Omega}\left(\nabla \cdot \mathbf{u}+\varphi_{0}\right) c^{\beta} \bar{\mu}^{\beta} \mathrm{d} \mathbf{x}, \quad \beta=+,-,
\end{aligned}
$$


then the problem $(2.2 \mathrm{a})-(2.2 \mathrm{~d})$ can be rewritten as follows:

Find $\left(\mathbf{u}, \mathbf{q}^{l}, \mathbf{q}_{\mathrm{tot}}^{+}, \mathbf{q}_{\mathrm{tot}}^{-}, \mu^{l}, \mu^{+}, \mu^{-}\right) \in \mathcal{V} \times H_{N}(\operatorname{div} ; \Omega) \times H_{N}(\operatorname{div} ; \Omega) \times H_{N}(\operatorname{div} ; \Omega) \times L^{2}(\Omega)$ $\times L^{2}(\Omega) \times L^{2}(\Omega)$ such that

$$
\frac{\mathrm{d}}{\mathrm{d} t} b\left(\mathbf{u}, \bar{\mu}^{l}\right): \begin{array}{ll}
a(\mathbf{u}, \overline{\mathbf{u}}) \\
\hdashline d^{l}\left(\mathbf{q}^{l}, \bar{\mu}^{l}\right) \\
d^{+}\left(\mathbf{q}_{\mathrm{tot}}^{+}, \bar{\mu}^{+}\right) \\
d^{-}\left(\mathbf{q}_{\mathrm{tot}}^{-}, \bar{\mu}^{-}\right) & =\frac{\mathrm{d}}{\mathrm{d} t} F_{2}^{+}\left(\bar{\mu}^{+}\right), \\
& =\frac{\mathrm{d}}{\mathrm{d} t} F_{2}^{-}\left(\bar{\mu}^{-}\right),
\end{array}
$$

for all test functions $\left(\overline{\mathbf{u}}, \overline{\mathbf{q}}^{l}, \overline{\mathbf{q}}_{\text {tot }}^{+}, \overline{\mathbf{q}}_{\text {tot }}^{-}, \bar{\mu}^{l}, \bar{\mu}^{+}, \bar{\mu}^{-}\right) \in \mathcal{V} \times H_{N}(\operatorname{div} ; \Omega) \times H_{N}(\operatorname{div} ; \Omega) \times H_{N}(\operatorname{div} ; \Omega) \times L^{2}(\Omega) \times L^{2}(\Omega) \times$ $L^{2}(\Omega)$ and $t>0$.

We shall use the implicit Euler scheme for time discretisation. Let $\Delta t$ be the time step and $\left(\mathbf{u}_{n}, \mathbf{q}_{n}^{l}, \mathbf{q}_{\mathrm{tot}, n}^{+}\right.$, $\left.\mathbf{q}_{\mathrm{tot}, n}^{-}, \mu_{n}^{l}, \mu_{n}^{+}, \mu_{n}^{-}\right)$the approximation of the solution vector $\left(\mathbf{u}, \mathbf{q}^{l}, \mathbf{q}_{\mathrm{tot}}^{+}, \mathbf{q}_{\mathrm{tot}}^{-}, \mu^{l}, \mu^{+}, \mu^{-}\right)$at $t=t_{n}=n \Delta t$. Then the system of equation (2.3) resulting from backward Euler is:

Find $\left(\mathbf{u}_{n}, \mathbf{q}_{n}^{l}, \mathbf{q}_{\text {tot }, n}^{+}, \mathbf{q}_{\text {tot }, n}^{-}, \mu_{n}^{l}, \mu_{n}^{+}, \mu_{n}^{-}\right) \in \mathcal{V} \times H_{N}(\operatorname{div} ; \Omega) \times H_{N}(\operatorname{div} ; \Omega) \times H_{N}(\operatorname{div} ; \Omega) \times L^{2}(\Omega)$ $\times L^{2}(\Omega) \times L^{2}(\Omega)$ such that

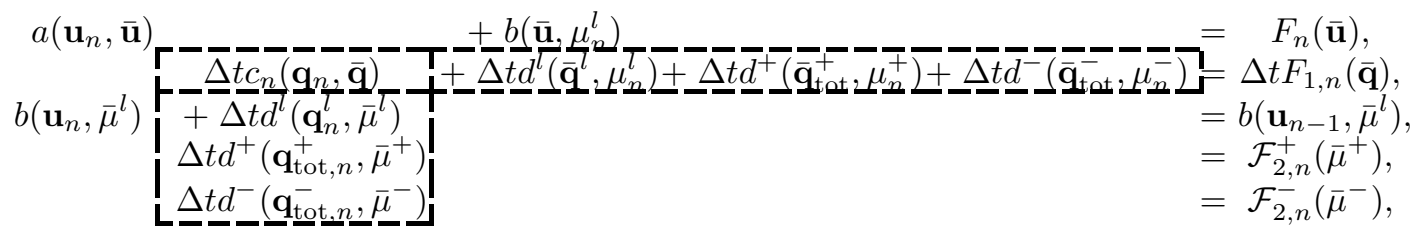

for all test functions $\left(\overline{\mathbf{u}}, \overline{\mathbf{q}}^{l}, \overline{\mathbf{q}}_{\text {tot }}^{+}, \overline{\mathbf{q}}_{\text {tot }}^{-}, \bar{\mu}^{l}, \bar{\mu}^{+}, \bar{\mu}^{-}\right) \in \mathcal{V} \times H_{N}(\operatorname{div} ; \Omega) \times H_{N}(\operatorname{div} ; \Omega) \times H_{N}(\operatorname{div} ; \Omega) \times L^{2}(\Omega) \times L^{2}(\Omega) \times$ $L^{2}(\Omega)$, where

where

$$
\mathcal{F}_{n}^{\beta}\left(\bar{\mu}^{\beta}\right)=F_{2, n}^{\beta}\left(\bar{\mu}^{\beta}\right)-F_{2, n-1}^{\beta}\left(\bar{\mu}^{\beta}\right), \quad \beta=+,-,
$$

$$
F_{2, n}^{\beta}\left(\bar{\mu}^{\beta}\right)=\int_{\Omega}\left(\nabla \cdot \mathbf{u}_{n}+\varphi_{0}\right) c_{n}^{\beta} \bar{\mu}^{\beta} \mathrm{d} \mathbf{x}, \quad \beta=+,-.
$$

Note that the dependency of $c_{n}\left(\mathbf{q}_{n}, \overline{\mathbf{q}}\right), F_{n}(\overline{\mathbf{u}}), F_{1, n}(\overline{\mathbf{q}})$ and $\mathcal{F}_{2, n}^{\beta}\left(\bar{\mu}^{+}\right)$to $n$ is because of $\varphi_{n}$ and $c_{n}^{\beta}$. Note that also we need some iteration procedure to solve the nonlinear system of equations. Let us define

$$
\begin{aligned}
\mathbb{A}_{n}(\mathbf{u}, \mathbf{q} ; \overline{\mathbf{u}}, \overline{\mathbf{q}}) & =a(\mathbf{u}, \overline{\mathbf{u}})+\Delta t c_{n}(\mathbf{q}, \overline{\mathbf{q}}), \\
\mathbb{B}(\mathbf{u}, \mathbf{q} ; \overline{\boldsymbol{\mu}}) & =b\left(\mathbf{u}, \bar{\mu}^{l}\right)+\Delta t d^{l}\left(\mathbf{q}^{l}, \bar{\mu}^{l}\right)+\Delta t d^{+}\left(\mathbf{q}_{\mathrm{tot}}^{+}, \bar{\mu}^{+}\right)+\Delta t d^{-}\left(\mathbf{q}_{\mathrm{tot}}^{-}, \bar{\mu}^{-}\right), \\
G_{1, n}(\overline{\mathbf{u}}, \overline{\mathbf{q}}) & =\left(F_{n}(\overline{\mathbf{u}}), \Delta t F_{1, n}(\overline{\mathbf{q}})\right)^{T}, G_{2, n}(\overline{\boldsymbol{\mu}})=\left(b\left(\mathbf{u}_{n-1}, \bar{\mu}^{l}\right), \mathcal{F}_{n}^{+}\left(\bar{\mu}^{+}\right), \mathcal{F}_{n}^{-}\left(\bar{\mu}^{-}\right)\right)^{T} .
\end{aligned}
$$

Then (2.4) is rewritten as

$$
\begin{aligned}
& \text { Find }\left(\mathbf{u}_{n}, \mathbf{q}_{n}, \boldsymbol{\mu}_{n}\right) \in \mathcal{V} \times\left(H_{N}(\operatorname{div} ; \Omega)\right)^{3} \times\left(L^{2}(\Omega)\right)^{3} \\
& \qquad \begin{aligned}
\mathbb{A}_{n}\left(\mathbf{u}_{n}, \mathbf{q}_{n} ; \overline{\mathbf{u}}, \overline{\mathbf{q}}\right)+\mathbb{B}\left(\overline{\mathbf{u}}, \overline{\mathbf{q}} ; \boldsymbol{\mu}_{n}\right) & =G_{1, n}(\overline{\mathbf{u}}), \\
\mathbb{B}\left(\mathbf{u}_{n}, \mathbf{q}_{n} ; \overline{\boldsymbol{\mu}}\right) & =G_{2, n}(\overline{\boldsymbol{\mu}}),
\end{aligned}
\end{aligned}
$$

for all test functions $(\overline{\mathbf{u}}, \overline{\mathbf{q}}, \overline{\boldsymbol{\mu}}) \in \mathcal{V} \times\left(H_{N}(\operatorname{div} ; \Omega)\right)^{3} \times\left(L^{2}(\Omega)\right)^{3}$.

Let skip the subscript $n$ in the above system and assume that $\varphi, c^{+}$and $c^{-}$are given.

Theorem 2.1. Consider the saddle point problem (2.8). Assume that $\varphi, c^{+}$and $c^{-}$are given, then 
(i) The bilinear form $\mathbb{A}$ is $\operatorname{ker}(\mathbb{B})$-elliptic, i.e., there exists a positive constant $\alpha\left(\varphi, c^{+}, c^{-}\right)$such that

$$
\mathbb{A}(\mathbf{u}, \mathbf{q} ; \mathbf{u}, \mathbf{q}) \geq \alpha\|(\mathbf{u}, \mathbf{q})\|_{1}, \quad \text { for all }(\mathbf{u}, \mathbf{q}) \in \operatorname{ker}(\mathbb{B}),
$$

where

$$
\|(\mathbf{u}, \mathbf{q})\|_{1}=\left(\|\mathbf{u}\|_{1}^{2}+\left\|\mathbf{q}^{l}\right\|_{\text {div } ; \Omega}^{2}+\left\|\mathbf{q}_{\text {tot }}^{+}\right\|_{\text {div } ; \Omega}^{2}+\left\|\mathbf{q}_{\text {tot }}^{-}\right\|_{\text {div } ; \Omega}^{2}\right)^{1 / 2} .
$$

(ii) The bilinear $\mathbb{B}$ satisfies the inf-sup condition

$$
\sup _{\mathbf{q} \in\left(H_{N}(\operatorname{div} ; \Omega)\right)^{3}} \frac{\mathbb{B}(\mathbf{u}, \mathbf{q} ; \boldsymbol{\mu})}{\|(\mathbf{u}, \mathbf{q})\|_{1}} \geq \beta\|\boldsymbol{\mu}\|_{0} \quad \text { for all } \boldsymbol{\mu} \in\left(L^{2}(\Omega)\right)^{3} .
$$

Proof. To prove the first part, take $(\mathbf{u}, \mathbf{q}) \in \operatorname{ker}(\mathbb{B})$, then we have

$$
\int_{\Omega} \nabla \cdot \mathbf{u} \bar{\mu}^{l} \mathrm{~d} \mathbf{x}+\Delta t\left(\int_{\Omega} \nabla \cdot \mathbf{q}^{l} \bar{\mu}^{l} \mathrm{~d} \mathbf{x}+\int_{\Omega} \nabla \cdot \mathbf{q}_{\mathrm{tot}}^{+} \bar{\mu}^{+} \mathrm{d} \mathbf{x}+\int_{\Omega} \nabla \cdot \mathbf{q}_{\mathrm{tot}}^{-} \bar{\mu}^{-} \mathrm{d} \mathbf{x}\right)=0,
$$

for all $\overline{\boldsymbol{\mu}}=\left(\bar{\mu}^{l}, \bar{\mu}^{+}, \bar{\mu}^{-}\right) \in\left(L^{2}(\Omega)\right)^{3}$. This results into

$$
\begin{aligned}
\nabla \cdot \mathbf{u}+\Delta t \nabla \cdot \mathbf{q}^{l} & =0, \\
\nabla \cdot \mathbf{q}_{\mathrm{tot}}^{+} & =0, \\
\nabla \cdot \mathbf{q}_{\mathrm{tot}}^{-} & =0 .
\end{aligned}
$$

It is easy to see that

$$
c(\mathbf{q}, \overline{\mathbf{q}})=\int_{\Omega} \mathbf{q} \mathbf{C}^{l \pm} \overline{\mathbf{q}} \mathrm{d} \mathbf{x}
$$

where

$$
\mathbf{C}^{l \pm}=\left(\begin{array}{ccc}
\frac{1}{K}+\frac{R T c^{+}}{D^{+} \varphi}+\frac{R T c^{-}}{D^{-} \varphi} & -\frac{R T}{D^{+} \varphi} & -\frac{R T}{D^{-} \varphi} \\
-\frac{R T}{D^{+} \varphi} & \frac{R T}{D^{+} \varphi c^{+}} & 0 \\
-\frac{R T}{D^{-} \varphi} & 0 & \frac{R T}{D^{-} \varphi c^{-}}
\end{array}\right) .
$$

But $\mathbf{C}^{l \pm}$ is symmetric positive definite. Indeed, if we define $\mathbf{N}=\operatorname{diag}\left(1, c^{+}, c^{-}\right)$, then we have

$$
\varphi^{2} \mathbf{N C}^{l \pm} \mathbf{N}=\left(\begin{array}{ccc}
\frac{\varphi^{2}}{K}+\frac{R T c^{+} \varphi}{D^{+}}+\frac{R T c^{-} \varphi}{D^{-}} & -\frac{R T c^{+} \varphi}{D^{+}} & -\frac{R T c^{-} \varphi}{D^{-}} \\
-\frac{R T c^{+} \varphi}{D^{+}} & \frac{R T c^{+} \varphi}{D^{+}} & 0 \\
-\frac{R T c^{-} \varphi}{D^{-}} & 0 & \frac{R T c^{-} \varphi}{D^{-}}
\end{array}\right) .
$$

In [10], Lemma 2.2, we proved that this matrix is symmetric positive definite. 
Now let us continue the proof of ker $(\mathbb{B})$-ellipticity of the bilinear form $\mathbb{A}$. Take $(\mathbf{u}, \mathbf{q}) \in \operatorname{ker}(\mathbb{B})$, then by using Korn's inequality (see for example [2]) and equations (2.9)-(2.12) we have

$$
\begin{aligned}
\mathbb{A}(\mathbf{u}, \mathbf{q} ; \mathbf{u}, \mathbf{q}) & =\int_{\Omega} 2 \mu_{s} \mathcal{E}(\mathbf{u}): \mathcal{E}(\mathbf{u})+\lambda_{s}(\nabla \cdot \mathbf{u})^{2} \mathrm{~d} \mathbf{x}+\Delta t \int_{\Omega}\left(\mathbf{q}^{l}, \mathbf{q}_{\text {tot }}^{+}, \mathbf{q}_{\text {tot }}^{-}\right) \mathbf{C}^{l \pm}\left(\begin{array}{c}
\overline{\mathbf{q}}^{l} \\
\overline{\mathbf{q}}_{\text {tot }}^{+} \\
\overline{\mathbf{q}}_{\text {tot }}^{-}
\end{array}\right) \\
& \geq 2 \mu_{s} c\|\mathbf{u}\|_{1}^{2}+\lambda_{s}\|\nabla \cdot \mathbf{u}\|_{0}^{2}+C \Delta t\left(\left\|\mathbf{q}^{l}\right\|_{0}^{2}+\left\|\mathbf{q}_{\text {tot }}^{+}\right\|_{0}^{2}+\left\|\mathbf{q}_{\text {tot }}^{-}\right\|_{0}^{2}\right) \\
& \geq 2 \mu_{s} c\|\mathbf{u}\|_{1}^{2}+\lambda_{s}(\Delta t)^{2}\left\|\nabla \cdot \mathbf{q}^{l}\right\|_{0}^{2}+C \Delta t\left(\left\|\mathbf{q}^{l}\right\|_{0}^{2}+\left\|\mathbf{q}_{\text {tot }}^{+}\right\|_{\text {div } ; \Omega}^{2}+\left\|\mathbf{q}_{\text {tot }}^{-}\right\|_{\text {div } ; \Omega}^{2}\right) \\
& \geq \alpha\|(\mathbf{u}, \mathbf{q})\|_{1},
\end{aligned}
$$

where $\alpha=\min \left(2 \mu_{s} c, \lambda_{s}(\Delta t)^{2}, C\right)$ and $C=C\left(\varphi, c^{+}, c^{-}\right)$.

To prove the second part (inf-sup condition), we restrict the supremum to a subset of functions $(\mathbf{0}, \mathbf{q})$,

$$
\Delta t \sup _{\mathbf{q} \in\left(H_{N}(\operatorname{div} ; \Omega)\right)^{3}} \frac{d^{l}\left(\mathbf{q}^{\mathbf{1}}, \mu^{l}\right)+d^{+}\left(\mathbf{q}_{\mathrm{tot}}^{+}, \mu^{+}\right)+d^{-}\left(\mathbf{q}_{\mathrm{tot}}^{-} \mu^{-}\right)}{\|\mathbf{q}\|_{\text {div } ; \Omega}} \geq \tilde{\beta}\|\boldsymbol{\mu}\|_{0},
$$

for all $\boldsymbol{\mu} \in\left(L^{2}(\Omega)\right)^{3}$. Easily it can be seen that the above supremum is greater or equal than

$$
\Delta t \sup _{\mathbf{q}^{l} \in H_{N}(\operatorname{div} ; \Omega)} \frac{d^{l}\left(\mathbf{q}^{\mathbf{l}}, \mu^{l}\right)}{\left\|\mathbf{q}^{l}\right\|_{\text {div } ; \Omega}}+\sup _{\mathbf{q}_{\text {tot }}^{+} \in H_{N}(\operatorname{div} ; \Omega)} \frac{d^{+}\left(\mathbf{q}_{\mathrm{tot}}^{+}, \mu^{+}\right)}{\left\|\mathbf{q}_{\mathrm{tot}}^{+}\right\|_{\text {div } ; \Omega}}+\sup _{\mathbf{q}_{\mathrm{tot}}^{-} \in H_{N}(\operatorname{div} ; \Omega)} \frac{d^{-}\left(\mathbf{q}_{\mathrm{tot}}^{-}, \mu^{-}\right)}{\left\|\mathbf{q}_{\mathrm{tot}}^{-}\right\|_{\text {div } ; \Omega}} .
$$

In fact, we split the inf-sup condition to three inf-sup condition for each component, liquid, cation and anion. To proceed the proof, the following lemma is needed.

Lemma 2.2. There exists a positive constant $C$ such that for all $q \in L^{2}(\Omega)$ there exists a function $\tilde{\mathbf{q}} \in$ $H_{N}(\operatorname{div} ; \Omega)$ satisfying

$$
-\nabla \cdot \tilde{\mathbf{q}}=q \quad \text { and } \quad\|\tilde{\mathbf{q}}\|_{\operatorname{div} ; \Omega} \leq C\|q\|_{0} .
$$

Proof. Let $q \in L^{2}(\Omega)$, then by the Lax-Miligram Theorem [1], Theorem 2.7.7, there exists a unique $\Phi \in H_{D}^{1}(\Omega)$ satisfying

$$
\left\{\begin{array}{lll}
-\Delta \Phi & =q & \text { in } \Omega \\
\nabla \Phi \cdot \mathbf{n} & =0 & \text { on } \Gamma_{p}^{N}
\end{array}\right.
$$

If we define $\tilde{\mathbf{q}}=\nabla \Phi$, then $\tilde{\mathbf{q}} \in H_{N}(\operatorname{div} ; \Omega)$ and we have

$$
\int_{\Omega} \tilde{\mathbf{q}} \nabla \bar{\Phi} \mathrm{d} \mathbf{x}=\int_{\Omega} q \bar{\Phi} \mathrm{d} \mathbf{x} \quad \text { for all } \bar{\Phi} \in H_{D}^{1}(\Omega) .
$$

By choosing $\bar{\Phi}=\Phi$, we have

$$
\|\tilde{\mathbf{q}}\|_{0}^{2}=\int_{\Omega} q \Phi \mathrm{d} \mathbf{x} \leq\|q\|_{0}\|\Phi\|_{0} \leq C(\Omega)\|q\|_{0}\|\nabla \Phi\|_{0}
$$

where the last inequality was derived by the Poincaré inequality [1], Proposition 5.3.5.

Let $\mu^{l} \in L^{2}(\Omega)$, then by above the lemma there exists a function $\tilde{\mathbf{q}} \in H_{N}(\operatorname{div} ; \Omega)$ such that $-\nabla \cdot \tilde{\mathbf{q}}=\mu^{l}$ and

$$
\|\tilde{\mathbf{q}}\|_{\text {div } ; \Omega} \leq C\left\|\mu^{l}\right\|_{0}
$$


for some constant $C$. Use the fact that

$$
d^{l}\left(\tilde{\mathbf{q}}, \mu^{l}\right)=-\int_{\Omega} \nabla \cdot \tilde{\mathbf{q}} \mu^{l} \mathrm{~d} \mathbf{x}=\int_{\Omega}\left(\mu^{l}\right)^{2} \mathrm{~d} \mathbf{x}=\left\|\mu^{l}\right\|_{0}^{2}
$$

therefore we have

$$
\Delta t \sup _{\mathbf{q} \in H_{N}(\operatorname{div} ; \Omega)} \frac{d^{l}\left(\mu^{l}, \mathbf{q}\right)}{\|\mathbf{q}\|_{\text {div } ; \Omega}} \geq \Delta t \frac{d^{l}\left(\mu^{l}, \tilde{\mathbf{q}}\right)}{\|\tilde{\mathbf{q}}\|_{\text {div } ; \Omega}} \geq \frac{\Delta t}{C}\left\|\mu^{l}\right\|_{0} .
$$

Therefore the inf-sup condition holds for all $\tilde{\beta}^{l} \leq \frac{\Delta t}{C}$. Similarly we have lower bounds $\tilde{\beta}^{+}$and $\tilde{\beta}^{-}$for the bilinear forms $d^{+}$and $d^{-}$, respectively. Thus

$$
\begin{aligned}
& \Delta t \quad \sup _{\mathbf{q} \in\left(H_{N}(\operatorname{div} ; \Omega)\right)^{3}} \frac{d^{l}\left(\mathbf{q}^{1}, \mu^{l}\right)+d^{+}\left(\mathbf{q}_{\text {tot }}^{+}, \mu^{+}\right)+d^{-}\left(\mathbf{q}_{\text {tot }}^{-}, \mu^{-}\right)}{\|\mathbf{q}\|_{\text {div } ; \Omega}} \geq \tilde{\beta}^{l}\left\|\mu^{l}\right\|_{0}+\tilde{\beta}^{+}\left\|\mu^{+}\right\|_{0}+\tilde{\beta}^{-}\left\|\mu^{-}\right\|_{0} \\
& \geq \quad \tilde{\beta}\left(\left\|\mu^{l}\right\|_{0}^{2}+\left\|\mu^{+}\right\|_{0}^{2}+\left\|\mu^{-}\right\|_{0}^{2}\right)^{1 / 2}=\tilde{\beta}\|\boldsymbol{\mu}\|_{0} .
\end{aligned}
$$

Therefore the inf-sup condition holds for all $\beta \leq \tilde{\beta}$. This proves the second part of the theorem.

Remark 2.3. Following the above theorem, we can conclude that given $\varphi, c^{+}$and $c^{-}$the solution for the system (2.8) exists and is unique. Moreover, one has the bounds

$$
\begin{gathered}
\|(\mathbf{u}, \mathbf{q})\|_{1} \leq \frac{1}{\alpha}\|F\|_{-1}+\left(1+\frac{\|\mathbb{A}\|}{\alpha}\right) \frac{1}{\beta}\|G\|_{-1}, \\
\|\boldsymbol{\mu}\|_{\left(L^{2}(\Omega)\right)^{3} \backslash \operatorname{ker}\left(\mathbb{B}^{*}\right)} \leq \frac{1}{\beta}\left(1+\frac{\|\mathbb{A}\|}{\alpha}\right)\|F\|_{-1}+\frac{\|\mathbb{A}\|}{\beta^{2}}\left(1+\frac{\|\mathbb{A}\|}{\alpha}\right)\|G\|_{-1},
\end{gathered}
$$

where

$$
\operatorname{ker}\left(\mathbb{B}^{*}\right)=\left\{\boldsymbol{\mu} \in \mathbf{L}^{2}(\Omega) \mid \mathbb{B}(\mathbf{u}, \mathbf{q} ; \boldsymbol{\mu})=0 \quad \text { for all }(\mathbf{u}, \mathbf{q}) \in \mathcal{V} \times H_{N}(\operatorname{div} ; \Omega)\right\}
$$

\subsection{A mixed finite element method}

Assume that $\Omega$ is a polygon $(n=2)$ or a polyhedron $(n=3)$. We denote by $\mathcal{T}_{h}$ a triangulation of $\bar{\Omega}$ by $n$-simplices $T$ of diameter not greater than $h$ ( $T$ is a triangle or rectangle for $n=2$, a tetrahedron or block for $n=3$ ), where

$$
\bar{\Omega}=\bigcup_{T \in \mathcal{T}_{h}} T .
$$


For the definition of a triangulation, see [3], p. 38, for example. We shall also use the notation:

- meas $T=$ the Euclidian measure of $T$ in $\mathbb{R}^{n}$ (geometric area if $n=2$, geometric volume if $n=3$ );

$-h_{T}=$ the diameter of $T$, which in case of a triangulation by simplices, is just the length of the longest edge;

$-\rho_{T}=$ the radius of the circle inscribed in $T$ if $n=2$, of the sphere inscribed in $T$ if $n=3$;

$-h=\max _{T \in \mathcal{T}_{h}} h_{T}$.

A family of triangulations $\left\{\mathcal{T}_{h}: h>0\right\}$ is said to be regular if $\inf _{h>0} h=0$ and $\inf _{h>0} \min _{T \in \mathcal{T}_{h}} \frac{\rho_{T}}{h_{T}}>0$.

Now in order to state a finite element formulation of the problem (2.3), it is necessary to define finite-dimensional subspaces of $\mathcal{V}, H_{N}(\operatorname{div} ; \Omega)$ and $L^{2}(\Omega)$.

Let $H\left(\operatorname{div} ; \mathcal{T}_{h}\right)$ be the space of square-integrable vectorial functions $\mathbf{q} \in \mathbf{L}^{2}(\Omega)$, whose divergences are square-integrable on every sub-domain $T \in \mathcal{T}_{h}$, i.e.

$$
H\left(\operatorname{div} ; \mathcal{T}_{h}\right)=\left\{\mathbf{q} \in \mathbf{L}^{2}(\Omega):\left.\mathbf{q}\right|_{T} \in H(\operatorname{div} ; T) \text { for all } T \in \mathcal{T}_{h}\right\},
$$

with norm

$$
\|\mathbf{q}\|_{\text {div; } ; \mathcal{T}_{h}}=\left(\|\mathbf{q}\|_{0}^{2}+\sum_{T \in \mathcal{T}_{h}}\left\|\left.\nabla \cdot \mathbf{q}\right|_{T}\right\|_{0}^{2}\right)^{1 / 2} .
$$

In order to define finite-dimensional basis functions for $H\left(\operatorname{div} ; \mathcal{T}_{h}\right)$, we need to define a local basis function on a reference element. Let $\hat{T}$ be the convex hull of $L$ suitably chosen points $\hat{\mathbf{x}}_{\ell}, \ell=1, \ldots, L$, that is

$$
\hat{T}=\left\{\hat{\mathbf{x}}=\sum_{1}^{L} \zeta_{\ell} \hat{\mathbf{x}}_{\ell}: 0 \leq \zeta_{\ell} \leq 1, \sum_{1}^{L} \zeta_{\ell}=1\right\} .
$$

Define the affine map

$$
\mathbf{x}=\mathbf{F}_{n}(\hat{\mathbf{x}})=\mathbf{B}_{n} \hat{\mathbf{x}}+\mathbf{b}_{n}, \quad \hat{\mathbf{x}} \in \hat{T},
$$

where $\mathbf{B}_{n} \in \mathbb{R}^{n \times n}$ such that $\operatorname{det} \mathbf{B}_{n}>0$, and $\mathbf{b}_{n} \in \mathbb{R}^{n}$.

Define $P^{1}(\hat{T})$ as the $L$-dimensional space of polynomials spanned by the basis functions $\hat{\varphi}_{i}, i=1, \ldots, L$, such that

$$
\hat{\varphi}_{i}\left(\hat{\mathbf{x}}_{j}\right)=\delta_{i j} .
$$

Now, define $P^{1}(T)$ as the $L$-dimensional space spanned by

$$
\varphi_{i}(\mathbf{x})=\hat{\varphi}_{i}(\hat{\mathbf{x}}), \quad i=1, \ldots, L .
$$

It is well known that

$$
\int_{T} \varphi_{i} \mathrm{~d} \mathbf{x}=\int_{\hat{T}} \hat{\varphi}_{i} \operatorname{det} \mathbf{B}_{n} \mathrm{~d} \hat{\mathbf{x}} .
$$

Also

$$
\nabla \varphi_{i}(\mathbf{x})=\mathbf{B}_{n}^{-T} \nabla \hat{\varphi}(\hat{\mathbf{x}}) \quad \text { for all } \hat{\mathbf{x}} \in \hat{T} .
$$

Since $\mathcal{V}$ consists of vectorial functions, the following subspaces are defined to approximate each component of functions in this space.

$$
\begin{aligned}
P_{-1}^{1}\left(\mathcal{T}_{h}\right) & =\left\{\varphi \in L^{2}(\Omega):\left.\varphi\right|_{T} \in P^{1}(T) \text { for all } T \in \mathcal{T}_{h}\right\} \\
P_{0}^{1}\left(\mathcal{T}_{h}\right) & =P_{-1}^{1}\left(\mathcal{T}_{h}\right) \cap H^{1}(\Omega), \\
P_{D}^{1}\left(\mathcal{T}_{h}\right) & =\left\{\varphi \in P_{0}^{1}\left(\mathcal{T}_{h}\right): \varphi=0 \text { on } \Gamma_{\mathbf{u}}^{D}\right\}
\end{aligned}
$$


Let $\hat{e}_{i}, i=1, \ldots, I$, be the edges $(n=2)$ or faces $(n=3)$ of $\hat{T}$, and $R T^{0}(\hat{T})$ be the $I$-dimensional space of linear vectorial functions $\hat{\mathbf{u}}$ on $\hat{T}$ such that $\mathbf{n}_{\hat{T}} \cdot \hat{\mathbf{u}}$ is constant on $\hat{e}_{i}, i=1, \ldots, I$. The basis functions for this space are $\hat{\mathbf{v}}_{i}, i=1, \ldots, I$, such that

$$
\int_{\hat{e}_{j}} \mathbf{n}_{\hat{T}} \cdot \hat{\mathbf{v}}_{i} \mathrm{~d} \hat{s}=\delta_{i j}, \quad i, j=1, \ldots, I .
$$

Define $R T^{0}(T)$ be the space spanned by the basis functions

$$
\mathbf{v}_{i}(\mathbf{x})=\left(\operatorname{det} \mathbf{B}_{n}\right)^{-1} \mathbf{B}_{n} \hat{\mathbf{v}}_{i}(\hat{\mathbf{x}}), \quad \hat{\mathbf{x}} \in \hat{T},
$$

that is,

$$
R T^{0}(T)=\left\{\left(\operatorname{det} \mathbf{B}_{n}\right)^{-1} \mathbf{B}_{n} \hat{\mathbf{u}}, \quad \hat{\mathbf{u}} \in R T^{0}(\hat{T})\right\} .
$$

Theorem 2.4. Let $\hat{\mathbf{u}} \in \mathbf{L}^{2}(\hat{T})$ and $\hat{\varphi} \in L^{2}(\hat{T})$.

$$
\mathbf{u}(\mathbf{x})=\left(\operatorname{det} \mathbf{B}_{n}\right)^{-1} \mathbf{B}_{n} \hat{\mathbf{u}}, \quad \varphi(\mathbf{x})=\hat{\varphi}(\hat{\mathbf{x}}),
$$

then the following equalities hold:

$$
\begin{aligned}
\int_{T} \mathbf{u} \cdot \nabla \varphi \mathrm{d} \mathbf{x} & =\int_{\hat{T}} \hat{\mathbf{u}} \cdot \nabla \hat{\varphi} \mathrm{d} \hat{\mathbf{x}}, \text { for all } \hat{\mathbf{u}} \in \mathbf{L}^{2}(\hat{T}), \hat{\varphi} \in H^{1}(\hat{T}), \\
\int_{T} \varphi \nabla \cdot \mathbf{u} \mathrm{d} \mathbf{x} & =\int_{\hat{T}} \hat{\varphi} \nabla \cdot \hat{\mathbf{u}} \mathrm{d} \hat{\mathbf{x}}, \text { for all } \hat{\varphi} \in L^{2}(\hat{T}), \hat{\mathbf{u}} \in H(\operatorname{div} ; \hat{T}), \\
\int_{\partial T} \varphi \mathbf{n}_{T} \cdot \mathbf{u} \mathrm{d} s & =\int_{\partial \hat{T}} \hat{\varphi} \mathbf{n}_{\hat{T}} \cdot \hat{\mathbf{u}} \mathrm{d} \hat{s}, \text { for all } \hat{\varphi} \in H^{1}(\hat{T}), \hat{\mathbf{u}} \in H(\operatorname{div} ; \hat{T}),
\end{aligned}
$$

where $\mathbf{n}_{T}$ and $\mathbf{n}_{\hat{T}}$ are the outward normals to $\partial T$ and $\partial \hat{T}$, respectively.

Proof. For a proof, see [16], Proposition II-5.2 and II-5.4.

From equation $(2.33)$ it follows that, if $\hat{\mathbf{u}} \in H(\operatorname{div} ; \hat{T})$, then $\mathbf{u} \in H(\operatorname{div} ; T)$ and

$$
\nabla \cdot \mathbf{u}(\mathbf{x})=\left(\operatorname{det} \mathbf{B}_{n}\right)^{-1} \nabla \cdot \hat{\mathbf{u}}(\hat{\mathbf{x}}) .
$$

Note by Theorem 2.4 and relation (2.30), for every $\mathbf{u} \in R T^{0}(T), \mathbf{n}_{T} \cdot \mathbf{u}$ is constant on the edges $(n=2)$, or faces $(n=3) e_{i}, i=1, \ldots, I$.

The details and an overview of possible choices for the reference element $\hat{T}$ and the assigned shape functions can be found in [9].

Using definitions (2.31), we define the Raviart-Thomas spaces

$$
\begin{aligned}
R T_{-1}^{0}\left(\mathcal{T}_{h}\right) & =\left\{\mathbf{u} \in \mathbf{L}^{2}(\Omega):\left.\mathbf{u}\right|_{T} \in R T^{0}(T) \text { for all } T \in \mathcal{T}_{h}\right\} \\
R T_{0}^{0}\left(\mathcal{T}_{h}\right) & =R T_{-1}^{0}\left(\mathcal{T}_{h}\right) \cap H(\operatorname{div} ; \Omega) \\
R T_{0, N}^{0}\left(\mathcal{T}_{h}\right) & =R T_{-1}^{0}\left(\mathcal{T}_{h}\right) \cap H_{N}(\operatorname{div} ; \Omega)
\end{aligned}
$$

Further, $\mu^{l}$ and $\mu^{\beta}, \beta=+,-$, in $L^{2}\left(\mathcal{T}_{h}\right)$ are approximated by piecewise constant functions. Therefore, let $M^{0}(T)$ be the one-dimensional space of constant scalar functions on $T$. Its basis function is $\psi$, where $\psi(\mathbf{x})=1$ for $\mathbf{x} \in T$.

Let $\mathbf{x}_{i}, i=1, \ldots, I$, be the numbered nodes of $\mathcal{T}_{h} ; \mathcal{E}_{h}$ be the collection of edges $(n=2)$ or faces $(n=3)$ of sub-domains $T \in \mathcal{T}_{h} ; e_{j}, j=1, \ldots, J$, be the numbered edges $(n=2)$ or faces $(n=3)$ of $\left\{e \in \mathcal{T}_{h}: e \not \subset \bar{\Gamma}_{p}^{D}\right\}$ and $T_{k}, k=1, \ldots, K$, be the numbered sub-domains of $\mathcal{T}_{h}$. 
The finite-dimensional space $R T_{0, N}^{0}\left(\mathcal{T}_{h}\right)$ is spanned by the linearly independent vectorial basis functions $\mathbf{v}_{i}$, $i=1, \ldots, J$, such that

$$
\int_{e_{j}} \mathbf{n}_{j} \cdot \mathbf{v}_{i} \mathrm{~d} s=\delta_{i j}, \quad i, j=1, \ldots, J
$$

where the normal vector $\mathbf{n}_{j}$ is the normal to $e_{j}$ pointing from $T_{k}$ to $T_{l}, k>l$, if $e_{j}=T_{k} \cap T_{l}$, and outward if $e_{j} \subset \Gamma_{p}^{D}$.

The multiplier space $M_{-1}^{0}\left(\mathcal{T}_{h}\right)$ is defined as

$$
M_{-1}^{0}\left(\mathcal{T}_{h}\right)=\left\{\lambda \in L^{2}(\Omega):\left.\lambda\right|_{T} \in M^{0}(T) \text { for all } T \in \mathcal{T}_{h}\right\} .
$$

Its basis functions are $\psi_{k}, k=1, \ldots, K$, such that

$$
\psi_{k}(\mathbf{x})=\delta_{k l}, \quad \mathbf{x} \in T_{l}, \quad k, l=1, \ldots, K .
$$

\subsection{Hybridization of the mixed method}

Fraeijs de Veubeke $[5,6]$ introduced a hybrid method for the mixed formulation in order to simplify the solution of the algebraic system that must be solved in the procedure. In [9] mixed-hybrid finite element discretisation is used to solve an important class of problems in mathematical physics which involves equations of the form

$$
\left\{\begin{array}{l}
\mathbf{q}=-\mathbf{A} \nabla \mu \\
\nabla \cdot \mathbf{q}=f
\end{array}\right.
$$

where $\mathbf{A}$ is a symmetric and uniformly positive definite second order tensor. It can be seen that mixed formulation of the above system leads to a saddle point problem. Using the hybridization technique, the mixed finite element method results in a system of linear equations with a sparse and symmetric positive definite coefficients matrix.

Despite to this fact, hybridization method reduces the number of degrees of freedom. Furthermore, in the computations we only have the inverse of element-wise block diagonal matrix.

In this section hybridization technique is used for four component model. First we introduce a hybridization procedure for the mixed formulation. This will simplify the solution of the algebraic system that must be solved in the procedure. The idea behind hybridization is to relax the continuity requirement for the variables $\mathbf{q}^{l}$ and $\mathbf{q}_{\mathrm{tot}}^{\beta}$ across the internal edges. This will require to enlarge the Raviart-Thomas space in which $\mathbf{q}^{l}$ and $\mathbf{q}_{\mathrm{tot}}^{\beta}$ are sought and to introduce Lagrange multipliers to enforce the continuity of the normal component of $\mathbf{q}^{l}$ and $\mathbf{q}_{\text {tot }}^{\beta}$ across the inter-element boundaries. Define

$$
\mathcal{E}_{h}^{\partial}=\left\{e \in \mathcal{E}_{h}: e \subset \Gamma\right\} .
$$

We assume that $\Gamma_{p}^{D}$ is the union of some $e \in \mathcal{E}_{h}^{\partial}$.

Let $\tilde{\mu}_{i n}^{l}$ and $\tilde{\mu}_{i n}^{\beta}$, both in $L^{2}(\Omega)$, be piecewise constant approximations of $\mu_{i n}^{l}$ and $\mu_{i n}^{\beta}$, such that

$$
\begin{aligned}
& \int_{e}\left(\tilde{\mu}_{i n}^{l}-\mu_{i n}^{l}\right) \mathrm{d} s=0 \text { for all } e \in \mathcal{E}_{h}^{\partial}, e \subset \Gamma_{p}^{D}, \\
& \int_{e}\left(\tilde{\mu}_{i n}^{\beta}-\mu_{i n}^{\beta}\right) \mathrm{d} s=0 \text { for all } e \in \mathcal{E}_{h}^{\partial}, e \subset \Gamma_{p}^{D}, \beta=+,-.
\end{aligned}
$$

The hybrid formulation will make use of Lagrangian multipliers belonging to the space $L^{2}\left(\mathcal{E}_{h}\right)$ that is defined to be the product space

$$
L^{2}\left(\mathcal{E}_{h}\right)=\prod_{T \in \mathcal{T}_{h}} L^{2}(\partial T)
$$


Recall that $M^{0}(e), e \in \mathcal{E}_{h}$, is the space of constants functions on $e$. Define the multiplier spaces

$$
\begin{aligned}
M_{-1}^{0}\left(\mathcal{E}_{h}\right) & =\left\{\lambda=\left(\lambda_{e}\right)_{e \in \mathcal{E}_{h}} \in H^{1 / 2}\left(\bigcup_{e \in \mathcal{E}_{h}} e\right): \lambda_{e} \in M^{0}(e) \text { for all } e \in \mathcal{E}_{h}\right\}, \\
M_{-1,0}^{0}\left(\mathcal{E}_{h}\right) & =\left\{\lambda \in M_{-1}^{0}\left(\mathcal{E}_{h}\right): \lambda=0 \quad \text { on } \quad \Gamma_{p}^{D}\right\}, \\
M_{-1, l}^{0}\left(\mathcal{E}_{h}\right) & =\left\{\lambda \in M_{-1}^{0}\left(\mathcal{E}_{h}\right): \lambda=\tilde{\mu}_{i n}^{l} \quad \text { on } \quad \Gamma_{p}^{D}\right\}, \\
M_{-1, \beta}^{0}\left(\mathcal{E}_{h}\right) & =\left\{\lambda \in M_{-1}^{0}\left(\mathcal{E}_{h}\right): \lambda=\tilde{\mu}_{i n}^{\beta} \quad \text { on } \quad \Gamma_{p}^{D}\right\}, \beta=+,-.
\end{aligned}
$$

The following lemma is an immediate consequence of the above definitions.

Lemma 2.5. Suppose $\mathbf{q} \in R T_{-1}^{0}\left(\mathcal{T}_{h}\right)$, then $\mathbf{q} \in R T_{0, N}^{0}\left(\mathcal{T}_{h}\right)$ if, and only if,

$$
\sum_{T \in \mathcal{T}_{h}} \int_{\partial T} \lambda \mathbf{n}_{T} \cdot \mathbf{q} \mathrm{d} s=0, \quad \text { for all } \lambda \in M_{-1,0}^{0}\left(\mathcal{E}_{h}\right) .
$$

\section{Mixed hybrid variational problem:}

A spatial semi-discrete approximation by considering the hybridization technique is as follow.

Find $\left(\mathbf{u}_{h}, \mathbf{q}_{h}^{l}, \mathbf{q}_{\text {tot }, h}^{+}, \mathbf{q}_{\text {tot }, h}^{-}, \mu_{h}^{l}, \mu_{h}^{+}, \mu_{h}^{-}, \lambda_{h}^{l}, \lambda_{h}^{+}, \lambda_{h}^{-}\right)(\cdot, t) \in\left(P_{D}^{1}\left(\mathcal{T}_{h}\right)\right)^{n} \times R T_{-1}^{0}\left(\mathcal{T}_{h}\right) \times R T_{-1}^{0}\left(\mathcal{T}_{h}\right) \times R T_{-1}^{0}\left(\mathcal{T}_{h}\right) \times$ $M_{-1}^{0}\left(\mathcal{T}_{h}\right) \times M_{-1}^{0}\left(\mathcal{T}_{h}\right) \times M_{-1}^{0}\left(\mathcal{T}_{h}\right) \times M_{-1, l}^{0}\left(\mathcal{E}_{h}\right) \times M_{-1,+}^{0}\left(\mathcal{E}_{h}\right) \times M_{-1,-}^{0}\left(\mathcal{E}_{h}\right)$, such that

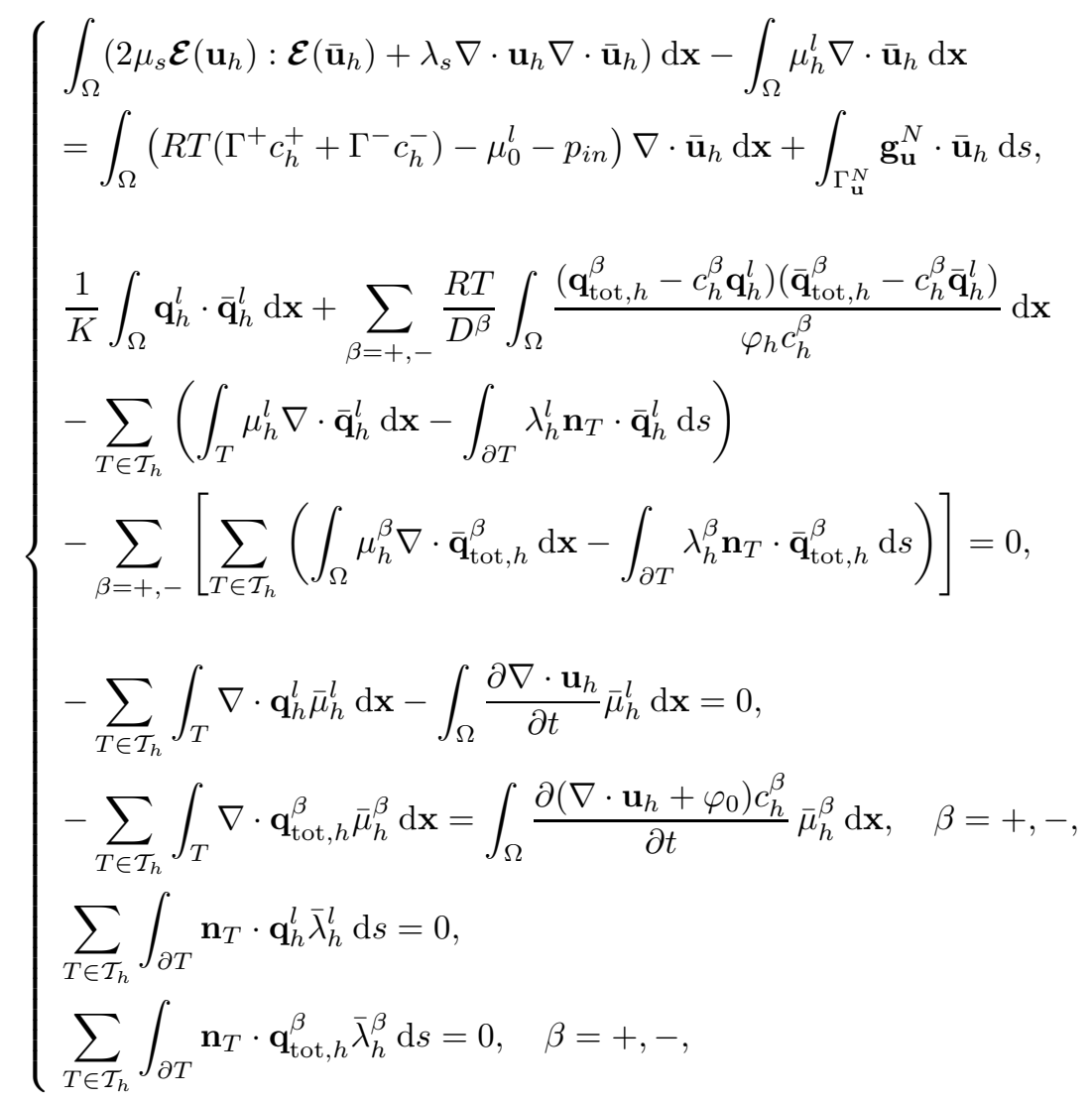

for all test functions $\left(\overline{\mathbf{u}}_{h}, \overline{\mathbf{q}}_{h}^{l}, \overline{\mathbf{q}}_{\text {tot }, h}^{+}, \mathbf{q}_{\mathrm{tot}, h}^{-}, \bar{\mu}_{h}^{l}, \bar{\mu}_{h}^{+}, \bar{\mu}_{h}^{-}, \bar{\lambda}_{h}^{l}, \bar{\lambda}_{h}^{+}, \bar{\lambda}_{h}^{-}\right) \in\left(P_{D}^{1}\left(\mathcal{T}_{h}\right)\right)^{n} \times R T_{-1}^{0}\left(\mathcal{T}_{h}\right) \times R T_{-1}^{0}\left(\mathcal{T}_{h}\right) \times$ $R T_{-1}^{0}\left(\mathcal{T}_{h}\right) \times M_{-1}^{0}\left(\mathcal{T}_{h}\right) \times M_{-1}^{0}\left(\mathcal{T}_{h}\right) \times M_{-1}^{0}\left(\mathcal{T}_{h}\right) \times M_{-1, D}^{0}\left(\mathcal{E}_{h}\right) \times M_{-1, D}^{0}\left(\mathcal{E}_{h}\right) \times M_{-1, D}^{0}\left(\mathcal{E}_{h}\right)$ and $t>0$ 
Note that in the above variational formulation the values for $\varphi_{h}$ and $c_{h}^{\beta}$ are calculated from the secondary equations mentioned in (1.1).

Let $\mathbf{x}_{i}, i=1, \ldots, I$, be numbered nodes of the triangulation $\left\{\mathbf{x} \in \mathcal{T}_{h}: \mathbf{x} \notin \Gamma_{\mathbf{u}}^{D}\right\} ; T_{k}, k=1, \ldots, K$, be numbered sub-domains of $\mathcal{T}_{h}$ and $e_{j}^{(T)}, j=1, \ldots, J^{(T)}$, be the edges $(n=2)$ or faces $(n=3)$ of $T$ for each $T \in \mathcal{T}_{h}$.

Recall that the finite-dimensional space $R T_{-1}^{0}\left(\mathcal{T}_{h}\right)$ is spanned by the linearly independent vectorial basis functions $\mathbf{v}_{j}^{(T)}, j=1, \ldots, J^{(T)}, T \in \mathcal{T}_{h}$, such that $\mathbf{v}_{j}^{(T)}$ has its support in $T$ and

$$
\int_{e_{j^{\prime}}^{(T)}} \mathbf{n}_{t} \cdot \mathbf{v}_{j}^{(T)} \mathrm{d} s=\delta_{j j^{\prime}}, \quad j, j^{\prime}=1, \ldots, J^{(S)}
$$

Thus a function $\mathbf{q} \in R T_{-1}^{0}\left(\mathcal{T}_{h}\right)$ has $J^{(T)}$ degrees of freedom per sub-domain $T \in \mathcal{T}_{h}$ and in total $J=\sum_{T \in \mathcal{T}_{h}} J^{(T)}$ degrees of freedom in $\mathcal{T}_{h}$. The degrees of freedom of $\mathbf{q}$ in the sub-domain $T \in \mathcal{T}_{h}$ are equal to

$$
\int_{e_{j}^{(T)}} \mathbf{n}_{T} \cdot \mathbf{q} \mathrm{d} s, \quad j=1, \ldots, J^{(T)} .
$$

Recall that the finite-dimensional space $M_{-1}^{0}\left(\mathcal{T}_{h}\right)$ is spanned by the linearly independent scalar basis functions $\psi_{k}, k=1, \ldots, K$, such that $(2.40)$ holds.

Let $e_{l}, l=1, \ldots, L$, be the numbered edges $(n=2)$ or faces $(n=3)$ of $\left\{e \in \mathcal{E}_{h}: e \not \subset \Gamma_{p}^{D}\right\}$. The finitedimensional space $M_{-1,0}^{0}\left(\mathcal{E}_{h}\right)$ is spanned by the linearly independent scalar basis functions $\eta_{l}, l=1, \ldots, L$, such that

$$
\eta_{l}(\mathbf{x})=\delta_{l l^{\prime}}, \quad \mathbf{x} \in e_{l^{\prime}}, \quad l, l^{\prime}=1, \ldots, L .
$$

Therefore a function $\lambda \in M_{-1,0}^{0}\left(\mathcal{E}_{h}\right)$ has one degree of freedom per edge $e_{l}, l=1, \ldots, L$, which is equal to its constant value on $e_{k}$.

Now by definition, functions $\mathbf{u}_{h}, \mu_{h}^{l}, \mu_{h}^{\beta}, \mathbf{q}_{h}^{l}, \mathbf{q}_{\mathrm{tot}, h}^{\beta}, \lambda_{h}^{l}$ and $\lambda_{h}^{\beta}$ are expressed as

$$
\begin{aligned}
\mathbf{u}_{h}(\mathbf{x}, t) & =\sum_{i=1}^{n I} \tilde{u}_{i}(t) \mathbf{w}_{i}(\mathbf{x}), \\
\mathbf{q}_{h}^{l}(\mathbf{x}, t) & =\sum_{j=1}^{J} \tilde{q}_{j}^{l}(t) \mathbf{v}_{j}(\mathbf{x}), \\
\mathbf{q}_{\mathrm{tot}, h}^{\beta}(\mathbf{x}, t) & =\sum_{j=1}^{J} \tilde{q}_{\mathrm{tot}, j}^{\beta}(t) \mathbf{v}_{j}(\mathbf{x}), \quad \beta=+,-, \\
\mu_{h}^{l}(\mathbf{x}, t) & =\sum_{k=1}^{K} \tilde{\mu}_{k}^{l}(t) \psi_{k}(\mathbf{x}), \\
\mu_{h}^{\beta}(\mathbf{x}, t) & =\sum_{k=1}^{K} \tilde{\mu}_{k}^{\beta}(t) \psi_{k}(\mathbf{x}), \quad \beta=+,-, \\
\lambda_{h}^{l}(\mathbf{x}, t) & =\lambda_{i n}^{l}+\sum_{\ell=1}^{L} \tilde{\lambda}_{\ell}^{l}(t) \eta_{l}(\mathbf{x}), \\
\lambda_{h}^{\beta}(\mathbf{x}, t) & =\lambda_{i n}^{\beta}+\sum_{\ell=1}^{L} \tilde{\lambda}_{\ell}^{\beta}(t) \eta_{l}(\mathbf{x}), \quad \beta=+,-,
\end{aligned}
$$


where $\lambda^{\beta}, \beta=l,+,-$ are defined by

$$
\lambda_{i n}^{\beta} \in M_{-1}^{0}\left(\mathcal{E}_{h}\right), \quad \begin{cases}\lambda_{i n}^{\beta}=\tilde{\mu}_{i n}^{\beta} & \text { on } \Gamma_{p}^{D}, \\ \lambda_{i n}^{\beta}=0 & \text { on } \bigcup_{e \in \mathcal{E}_{h}} e \backslash \Gamma_{p}^{D} .\end{cases}
$$

Substituting this in the mixed-hybrid variational formulation gives

$$
\mathfrak{A}\left(\varphi_{h}, c_{h}^{+}, c_{h}^{-}\right) \frac{\mathrm{d} \mathbf{y}}{\mathrm{d} t}+\mathfrak{B}\left(\varphi_{h}, c_{h}^{+}, c_{h}^{-}\right) \mathbf{y}=\mathfrak{F}\left(\varphi_{h}, c_{h}^{+}, c_{h}^{-}\right)+\frac{\mathrm{d} \mathfrak{G}}{\mathrm{d} t}\left(\varphi_{h}, c_{h}^{+}, c_{h}^{-}\right),
$$

where $\boldsymbol{\mathfrak { A }}\left(\varphi_{h}, c_{h}^{+}, c_{h}^{-}\right)$and $\mathfrak{B}\left(\varphi_{h}, c_{h}^{+}, c_{h}^{-}\right)$are

$$
\mathfrak{A}\left(\varphi_{h}, c_{h}^{+}, c_{h}^{-}\right)_{i j}= \begin{cases}\mathbf{B}^{T}, & i=5, j=1, \\ 0, & i \neq 5, j \neq 1,\end{cases}
$$

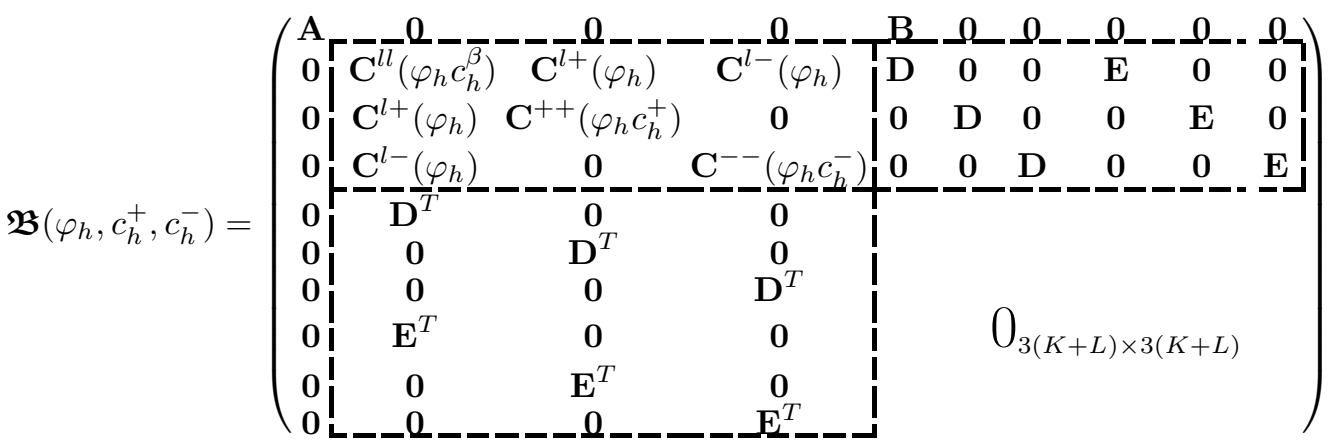

respectively, and

$$
\begin{aligned}
\mathbf{y} & =\left[\tilde{\mathbf{u}}, \tilde{\mathbf{q}}^{l}, \tilde{\mathbf{q}}_{\mathrm{tot}}^{+}, \tilde{\mathbf{q}}_{\mathrm{tot}}^{-}, \tilde{\boldsymbol{\mu}}^{l}, \tilde{\boldsymbol{\mu}}^{+}, \tilde{\boldsymbol{\mu}}^{-}, \tilde{\boldsymbol{\lambda}}^{l}, \tilde{\boldsymbol{\lambda}}^{+}, \tilde{\boldsymbol{\lambda}}^{-}\right]^{T}, \\
\mathfrak{F}\left(\varphi_{h}, c_{h}^{+}, c_{h}^{-}\right) & =\left[\mathbf{F}, \mathbf{F}_{1}^{l}, \mathbf{F}_{1}^{+}, \mathbf{F}_{1}^{-}, \mathbf{0}, \mathbf{0}, \mathbf{0}, \mathbf{0}, \mathbf{0}, \mathbf{0}\right]^{T} \\
\mathfrak{G}\left(\varphi_{h}, c_{h}^{+}, c_{h}^{-}\right) & =\left[\mathbf{0}, \mathbf{0}, \mathbf{0}, \mathbf{0}, \mathbf{0}, \mathbf{F}_{2}^{+}, \mathbf{F}_{2}^{-}, \mathbf{0}, \mathbf{0}, \mathbf{0}\right]^{T} .
\end{aligned}
$$

In the above formulations,

$$
\begin{aligned}
\tilde{\mathbf{u}} & =\left[\tilde{u}_{1}, \ldots, \tilde{u}_{n I}\right]^{T}, \\
\tilde{\mathbf{q}} & =\left[\tilde{q}_{1}, \ldots, \tilde{q}_{J}\right]^{T}, \\
\tilde{\mathbf{q}}_{\mathrm{tot}}^{\beta} & =\left[\tilde{q}_{\mathrm{tot}, 1}^{\beta}, \cdots, \tilde{q}_{\mathrm{tot}, J}^{\beta}\right]^{T}, \quad \beta=+,-, \\
\tilde{\boldsymbol{\mu}}^{l} & =\left[\tilde{\mu}_{1}^{l}, \ldots, \tilde{\mu}_{K}^{l}\right]^{T}, \\
\tilde{\boldsymbol{\mu}}^{\beta} & =\left[\tilde{\mu}_{1}^{\beta}, \ldots, \tilde{\mu}_{K}^{\beta}\right]^{T}, \quad \beta=+,-, \\
\tilde{\boldsymbol{\lambda}}^{l} & =\left[\tilde{\lambda}_{1}^{l}, \ldots, \tilde{\lambda}_{L}^{l}\right]^{T}, \\
\tilde{\boldsymbol{\lambda}}^{\beta} & =\left[\tilde{\lambda}_{1}^{\beta}, \ldots, \tilde{\lambda}_{L}^{\beta}\right]^{T}, \quad \beta=+,-,
\end{aligned}
$$




$$
\begin{aligned}
\mathbf{A}_{i j} & =\int_{\Omega}\left(2 \mu_{s} \mathcal{E}\left(\mathbf{w}_{i}\right): \mathcal{E}\left(\mathbf{w}_{j}\right)+\lambda_{s} \nabla \cdot \mathbf{w}_{i} \nabla \cdot \mathbf{w}_{j}\right) \mathrm{d} \mathbf{x}, \\
\mathbf{B}_{i j} & =-\int_{T_{j}} \nabla \cdot \mathbf{w}_{i} \mathrm{~d} \mathbf{x}, \\
\mathbf{C}_{i j}^{l l}\left(\varphi_{h} c_{h}^{\beta}\right) & =\frac{1}{K} \int_{\Omega} \mathbf{v}_{i} \cdot \mathbf{v}_{j} \mathrm{~d} \mathbf{x}+\sum_{\beta=+,-} \frac{R T}{D^{\beta}} \int_{\Omega} \frac{c_{h}^{\beta} \mathbf{v}_{i} \cdot \mathbf{v}_{j}}{\varphi_{h}} \mathrm{~d} \mathbf{x}, \\
\mathbf{C}_{i j}^{l \beta}\left(\varphi_{h}\right) & =-\frac{R T}{D^{\beta}} \int_{\Omega} \frac{\mathbf{v}_{i} \cdot \mathbf{v}_{j}}{\varphi_{h}} \mathrm{~d} \mathbf{x}, \quad \beta=+,-, \\
\mathbf{C}^{\beta \beta}\left(\varphi_{h} c_{h}^{\beta}\right) & =\frac{R T}{D^{\beta}} \int_{\Omega} \frac{\mathbf{v}_{i} \cdot \mathbf{v}_{j}}{\varphi_{h} c_{h}^{\beta}} \mathbf{x}, \quad \beta=+,- \\
\mathbf{D}_{i j} & =-\int_{T_{j}} \nabla \cdot \mathbf{v}_{i} \mathrm{~d} \mathbf{x}, \\
\mathbf{E}_{i k} & =\int_{e_{k}} \mathbf{n}_{i} \cdot \mathbf{v}_{i} \mathrm{~d} s, \\
\mathbf{F}_{i} & =\int_{\Omega}\left(R T\left(\Gamma^{+} c_{h}^{+}+\Gamma^{-} c_{h}^{-}\right)-\mu_{0}^{l}-p_{i n}\right) \nabla \cdot \mathbf{w}_{i} \mathrm{~d} \mathbf{x}+\int_{\Gamma_{\mathbf{u}}^{N}} \mathbf{g}_{\mathbf{u}}^{N} \cdot \mathbf{w}_{i} \mathrm{~d} s \\
\left(\mathbf{F}_{1}^{l}\right)_{i} & =-\int_{\Gamma} \tilde{\mu}_{i n}^{l} \mathbf{n} \cdot \mathbf{v}_{i} \mathrm{~d} s, \\
\left(\mathbf{F}_{1}^{\beta}\right)_{i} & =-\int_{\Gamma} \tilde{\mu}_{i n}^{\beta} \mathbf{n} \cdot \mathbf{v}_{i} \mathrm{~d} s, \quad \beta=+,- \\
\left(\mathbf{F}_{2}^{\beta}\right)_{i} & =\int_{T_{i}}\left(\nabla \cdot \mathbf{u}_{h}+\varphi_{0}\right) c_{h}^{\beta} \mathrm{d} \mathbf{x}, \quad \beta=+,-
\end{aligned}
$$

Remark 2.6. From now in our notations, we omit the dependencies of the matrix functions to $\varphi_{h}, c_{h}^{+}, c_{h}^{-}$. We keep in mind that this is just to make our formulas more readable.

Define

$$
\mathfrak{C}=\left(\begin{array}{ccc}
\mathbf{C}^{l l} & \mathbf{C}^{l+} & \mathbf{C}^{l-} \\
\mathbf{C}^{l+} & \mathbf{C}^{++} & \mathbf{0} \\
\mathbf{C}^{l-} & \mathbf{0} & \mathbf{C}^{--}
\end{array}\right), \quad \mathfrak{D}=\left(\begin{array}{ccc}
\mathbf{D} & \mathbf{0} & \mathbf{0} \\
\mathbf{0} & \mathbf{D} & \mathbf{0} \\
\mathbf{0} & \mathbf{0} & \mathbf{D}
\end{array}\right), \quad \mathfrak{E}=\left(\begin{array}{ccc}
\mathbf{E} & \mathbf{0} & \mathbf{0} \\
\mathbf{0} & \mathbf{E} & \mathbf{0} \\
\mathbf{0} & \mathbf{0} & \mathbf{E}
\end{array}\right)
$$

Theorem 2.7. $(\mathfrak{D} \mid \mathfrak{E})^{T} \mathfrak{C}^{-1}(\mathfrak{D} \mid \mathfrak{E})$ is a symmetric positive-definite matrix.

Proof. $\mathfrak{C}$ is symmetric positive-definite and $(\mathfrak{D} \mid \mathfrak{E})^{T} \mathfrak{C}^{-1}(\mathfrak{D} \mid \mathfrak{E})$ is symmetric positive semi-definite. To prove the positive-definiteness, we need to show that $(\mathfrak{D} \mid \mathfrak{E})$ has full rank. Let

$$
\begin{aligned}
& \mu_{h}^{\beta}(\mathbf{x}, t)= \sum_{k=1}^{K} \tilde{\mu}_{k}^{\beta}(t) \psi_{k}(\mathbf{x}) \in M_{-1}^{0}\left(\mathcal{T}_{h}\right), \quad \tilde{\boldsymbol{\mu}}^{\beta}=\left[\tilde{\mu}_{1}^{\beta}, \ldots, \tilde{\mu}_{K}^{\beta}\right]^{T}, \quad \beta=l,+,-, \\
& \lambda_{h}^{\beta}(\mathbf{x}, t)=\sum_{l=1}^{L} \tilde{\lambda}_{\beta}^{l}(t) \eta_{l}(\mathbf{x}) \in M_{-1}^{0}\left(\mathcal{E}_{h}\right), \quad \tilde{\boldsymbol{\lambda}}^{\beta}=\left[\tilde{\lambda}_{1}^{\beta}, \ldots, \tilde{\lambda}_{L}^{\beta}\right]^{T}, \quad \beta=l,+,-. \\
&(\mathfrak{D} \mid \mathfrak{E})\left[\tilde{\boldsymbol{\mu}}^{l}, \tilde{\boldsymbol{\mu}}_{,}^{+} \tilde{\boldsymbol{\mu}}^{-}, \tilde{\boldsymbol{\lambda}}^{l}, \tilde{\boldsymbol{\lambda}}^{+}, \tilde{\boldsymbol{\lambda}}^{-}\right]^{T}=\mathbf{0}, \quad \text { then } \quad\left\{\begin{array}{l}
\mathbf{D} \tilde{\boldsymbol{\mu}}^{l}+\mathbf{E} \tilde{\boldsymbol{\lambda}}^{l}=\mathbf{0}, \\
\mathbf{D} \tilde{\boldsymbol{\mu}}^{+}+\mathbf{E} \tilde{\boldsymbol{\lambda}}^{+}=\mathbf{0}, \\
\mathbf{D} \tilde{\boldsymbol{\mu}}^{-}+\mathbf{E} \tilde{\boldsymbol{\lambda}}^{-}=\mathbf{0} .
\end{array}\right.
\end{aligned}
$$


Since

$$
\begin{aligned}
& \mathbf{D} \tilde{\mu}^{l}+\mathbf{E} \tilde{\lambda}^{l}=0 \Leftrightarrow V^{T}\left(\mathbf{D} \tilde{\mu}^{l}+\mathbf{E} \tilde{\lambda}^{l}\right)=0 \quad \text { for all } V \in \mathbb{R}^{J} \\
\Leftrightarrow & \sum_{T \in \mathcal{T}_{h}}\left(\int_{T} \mu_{h}^{l} \nabla \cdot \mathbf{v}_{h} \mathrm{~d} \mathbf{x}-\int_{\partial \mathrm{T}} \lambda_{\mathrm{h}}^{l} \mathbf{n}_{\mathrm{T}} \cdot \mathbf{v}_{\mathrm{h}} \mathrm{ds}\right)=0 \quad \text { for all } \mathbf{v}_{h} \in R T_{-1}^{0}\left(\mathcal{T}_{h}\right) \\
\Leftrightarrow & \sum_{T \in \mathcal{T}_{h}} \int_{\partial T}\left(\mu_{h}^{l}-\lambda_{h}^{l}\right) \mathbf{n}_{T} \cdot \mathbf{v}_{h} \mathrm{~d} s=0 \quad \text { for all } \mathbf{v}_{h} \in R T_{-1}^{0}\left(\mathcal{T}_{h}\right) \\
\Leftrightarrow & \mu_{h}^{l}=\lambda_{h}^{l} \text { on } \partial T \quad \text { for all } T \in \mathcal{T}_{h} \Leftrightarrow \mu_{h}^{l}=0 \text { in } \Omega, \quad \lambda_{h}^{l}=0 \text { on } \bigcup_{e \in \mathcal{E}_{h}} e \\
\Leftrightarrow & \tilde{\boldsymbol{\mu}}^{l}=\mathbf{0}, \quad \tilde{\boldsymbol{\lambda}}^{l}=\mathbf{0} .
\end{aligned}
$$

Similarly $\tilde{\boldsymbol{\mu}}^{\beta}=\mathbf{0}$ and $\tilde{\boldsymbol{\lambda}}^{\beta}=\mathbf{0}$ for $\beta=+,-$. Therefore we have

$$
\left(\mathfrak{D} \Lambda_{1}+\mathfrak{E} \Lambda_{2}\right)^{T} \mathfrak{C}^{-1}\left(\mathfrak{D} \Lambda_{1}+\mathfrak{E} \Lambda_{2}\right)>0
$$

for all $\left(\boldsymbol{\Lambda}_{1}, \boldsymbol{\Lambda}_{2}\right) \in \mathbb{R}^{3(K+L)} \backslash\{\mathbf{0}\}$.

In the sequel the hybridization technique will be proceeded. Note that the system (2.50) can be considered as

$$
\left\{\begin{array}{c}
\mathbf{A} \tilde{\mathbf{u}}+\mathbf{B} \tilde{\boldsymbol{\mu}}^{l}=\mathbf{F}, \\
\mathfrak{C}\left(\begin{array}{c}
\tilde{\mathbf{q}}^{l} \\
\tilde{\mathbf{q}}_{\text {tot }}^{+} \\
\tilde{\mathbf{q}}_{\mathrm{tot}}^{-}
\end{array}\right)+(\mathfrak{D} \mid \mathfrak{E})\left(\begin{array}{c}
\tilde{\boldsymbol{\mu}}^{l} \\
\tilde{\boldsymbol{\mu}}^{+} \\
\tilde{\boldsymbol{\mu}}^{-} \\
\tilde{\boldsymbol{\lambda}}^{l} \\
\tilde{\boldsymbol{\lambda}}^{+} \\
\tilde{\boldsymbol{\lambda}}^{-}
\end{array}\right)=\left(\begin{array}{c}
\mathbf{0} \\
\mathbf{0} \\
\mathbf{0}
\end{array}\right), \\
\left(\begin{array}{c}
\mathbf{B}^{T} \\
\mathbf{0} \\
\mathbf{0} \\
\mathbf{0} \\
\mathbf{0} \\
\mathbf{0}
\end{array}\right) \frac{\mathrm{d}}{\mathrm{d} t} \tilde{\mathbf{u}}+(\mathfrak{D} \mid \mathfrak{E})^{T}\left(\begin{array}{c}
\tilde{\mathbf{q}}^{l} \\
\tilde{\mathbf{q}}_{\mathrm{tot}}^{+} \\
\tilde{\mathbf{q}}_{\mathrm{tot}}^{-}
\end{array}\right)=\frac{\mathrm{d}}{\mathrm{d} t}\left(\begin{array}{c}
\mathbf{0} \\
\mathbf{F}_{2}^{+} \\
\mathbf{F}_{2}^{-} \\
\mathbf{0} \\
\mathbf{0} \\
\mathbf{0}
\end{array}\right) .
\end{array}\right.
$$

In the system (2.54) $\mathfrak{C}$ is symmetric positive definite matrix and can be inverted at the finite element level (recall that $\mathbf{q}^{l}$ and $\mathbf{q}_{\text {tot }}^{\beta}$ are in the space $R T_{-1}^{0}\left(\mathcal{T}_{h}\right)$ ). Therefore the second equation in (2.54) implies

$$
\left(\begin{array}{c}
\tilde{\mathbf{q}}^{l} \\
\tilde{\mathbf{q}}_{\text {tot }}^{+} \\
\tilde{\mathbf{q}}_{\text {tot }}^{-}
\end{array}\right)=-\mathfrak{C}^{-1}(\mathfrak{D} \mid \mathfrak{E})\left(\begin{array}{c}
\tilde{\boldsymbol{\mu}}^{l} \\
\tilde{\boldsymbol{\mu}}^{+} \\
\tilde{\boldsymbol{\mu}}^{-} \\
\tilde{\lambda}^{l} \\
\tilde{\lambda}^{+} \\
\tilde{\lambda}^{-}
\end{array}\right)
$$


Using (2.55) and the third equation in (2.54) we get

$$
\left(\begin{array}{c}
\mathbf{B}^{T} \\
\mathbf{0} \\
\mathbf{0} \\
\mathbf{0} \\
\mathbf{0} \\
\mathbf{0}
\end{array}\right) \mathrm{d} \tilde{\mathrm{d} t} \tilde{\mathbf{u}}-(\mathfrak{D} \mid \mathfrak{E})^{T} \mathfrak{C}^{-1}(\mathfrak{D} \mid \mathfrak{E})\left(\begin{array}{c}
\tilde{\boldsymbol{\mu}}^{l} \\
\tilde{\boldsymbol{\mu}}^{+} \\
\tilde{\boldsymbol{\mu}}^{-} \\
\tilde{\boldsymbol{\lambda}}^{l} \\
\tilde{\boldsymbol{\lambda}}^{+} \\
\tilde{\boldsymbol{\lambda}}^{-}
\end{array}\right)=\frac{\mathrm{d}}{\mathrm{d} t}\left(\begin{array}{c}
\mathbf{0} \\
\mathbf{F}_{2}^{+} \\
\mathbf{F}_{2}^{-} \\
\mathbf{0} \\
\mathbf{0} \\
\mathbf{0}
\end{array}\right)
$$

In the next step we solve the above equation for $\left[\tilde{\boldsymbol{\mu}}^{l}, \tilde{\boldsymbol{\mu}}^{+}, \tilde{\boldsymbol{\mu}}^{-}\right]^{T}$. To do this, we need to state the following lemma.

Lemma 2.8. $\mathfrak{D}^{T} \mathfrak{C}^{-1} \mathfrak{D}$ is a symmetric positive definite matrix.

Proof. Since $\mathfrak{C}$ is symmetric positive definite matrices, therefore $\mathfrak{D}^{T} \mathfrak{C}^{-1} \mathfrak{D}$ is symmetric positive semi-definite. In order to prove that this matrix is positive definite, we need to find the null spaces of it.

Let $\mathfrak{D} \tilde{\mu}^{\beta}=0, \beta=l,+,-$, then

$$
\sum_{T \in \mathcal{T}_{h}} \int_{T} \mu_{h}^{\beta} \nabla \cdot \mathbf{v}_{h} \mathrm{~d} \mathbf{x}=0 \text { for all } \mathbf{v}_{h} \in R T_{-1}^{0}\left(\mathcal{T}_{h}\right), \quad \beta=l,+,-
$$

$\mathbf{v}_{h}$ is assumed to be continuous inside each element $T$, therefore the above summation will reduce to each element $T$. With an appropriate choice of $\mathbf{v}_{h}$, we can take $\nabla \cdot \mathbf{v}_{h}$ to be equal to 1 in $T$, therefore we have

$$
\int_{T} \mu_{h}^{\beta} \mathrm{d} \mathbf{x}=0 \quad \text { for all } T \in \mathcal{T}_{h}, \quad \beta=l,+,-.
$$

Hence

$$
\left.\mu_{h}^{\beta}\right|_{T}=0 \quad \text { for all } T \in \mathcal{T}_{h}, \quad \beta=l,+,- \text {. }
$$

This results in the symmetric positive definiteness of $\mathfrak{D}^{T} \mathfrak{C}^{-1} \mathfrak{D}$.

Remark 2.9. It can be seen that $\mathfrak{D}^{T} \mathfrak{C}^{-1} \mathfrak{D}$ is similar to a block diagonal matrix. In fact, we need to define an appropriate permutation matrix. Define $\mathbf{P}$ after reordering the rows of an identity matrix $\mathbf{I}_{3 L \times 3 L}$ based on the vector $(1, L+1,2 L+1,2, L+2,2 L+2, \ldots, L, 2 L, 3 L)$. Then it can be seen that the matrix $\mathbf{P}\left(\mathfrak{D}^{T} \mathfrak{C}^{-1} \mathfrak{D}\right) \mathbf{P}^{-1}$ is a block diagonal matrix and has $K$ blocks which are $3 \times 3$ matrices and the inverse is block diagonal too. This will imply that the inverse of $\mathfrak{D}^{T} \mathfrak{C}^{-1} \mathfrak{D}$ has the same pattern as $\mathfrak{D}^{T} \mathfrak{C}^{-1} \mathfrak{D}$.

Now by applying the above lemma and using equation (2.56) $\left[\tilde{\boldsymbol{\mu}}^{l}, \tilde{\boldsymbol{\mu}}^{+}, \tilde{\boldsymbol{\mu}}^{-}\right]^{T}$ can be expressed as

$$
\begin{aligned}
\left(\begin{array}{c}
\tilde{\boldsymbol{\mu}}^{l} \\
\tilde{\boldsymbol{\mu}}^{+} \\
\tilde{\boldsymbol{\mu}}^{-}
\end{array}\right) & =\left(\mathfrak{D}^{T} \mathfrak{C}^{-1} \mathfrak{D}\right)^{-1}\left[-\mathfrak{D}^{T} \mathfrak{C}^{-1} \mathfrak{E}\left(\begin{array}{c}
\tilde{\boldsymbol{\lambda}}^{l} \\
\tilde{\boldsymbol{\lambda}}^{+} \\
\tilde{\boldsymbol{\lambda}}^{-}
\end{array}\right)\right. \\
& \left.+\left(\begin{array}{c}
\mathbf{B}^{T} \\
\mathbf{0} \\
\mathbf{0}
\end{array}\right) \frac{\mathrm{d}}{\mathrm{d} t} \tilde{\mathbf{u}}-\frac{\mathrm{d}}{\mathrm{d} t}\left(\begin{array}{c}
\mathbf{0} \\
\mathbf{F}_{2}^{+} \\
\mathbf{F}_{2}^{-}
\end{array}\right)\right]
\end{aligned}
$$


If we substitute (2.57) in the system (2.56), then this system reduces to

$$
\left(\begin{array}{c}
\mathbf{A} \tilde{\mathbf{u}} \\
\mathbf{0}
\end{array}\right)+\left(\begin{array}{cc}
\mathfrak{A}_{1} & \mathfrak{A}_{2} \\
\mathfrak{A}_{2}^{T} & -\mathfrak{A}_{3}
\end{array}\right)\left(\begin{array}{c}
\frac{\mathrm{d}}{\mathrm{d} t} \tilde{\mathbf{u}} \\
\tilde{\boldsymbol{\lambda}}^{l} \\
\tilde{\boldsymbol{\lambda}}^{+} \\
\tilde{\boldsymbol{\lambda}}^{-}
\end{array}\right)=\left(\begin{array}{c}
\mathbf{F}_{1} \\
\mathbf{0}
\end{array}\right)+\left(\begin{array}{c}
\mathfrak{F}_{1} \\
\mathfrak{F}_{2}
\end{array}\right) \frac{\mathrm{d}}{\mathrm{d} t}\left(\begin{array}{c}
\mathbf{0} \\
\mathbf{F}_{2}^{+} \\
\mathbf{F}_{2}^{-}
\end{array}\right)
$$

where $\boldsymbol{\mathfrak { A }}_{1}, \boldsymbol{\mathfrak { A }}_{1}$ and $\boldsymbol{\mathfrak { A }}_{3}$ are

$$
\begin{aligned}
& \mathfrak{A}_{1}=\left(\begin{array}{lll}
\mathbf{B} & \mathbf{0} & \mathbf{0}
\end{array}\right)\left(\mathfrak{D}^{T} \mathfrak{C}^{-1} \mathfrak{D}\right)^{-1}\left(\begin{array}{c}
\mathbf{B}^{T} \\
\mathbf{0} \\
\mathbf{0}
\end{array}\right), \\
& \mathfrak{A}_{2}=-\left(\begin{array}{ccc}
\mathbf{B} & \mathbf{0} & \mathbf{0}
\end{array}\right)\left(\mathfrak{D}^{T} \mathfrak{C}^{-1} \mathfrak{D}\right)^{-1}\left(\mathfrak{D}^{T} \mathfrak{C}^{-1} \mathfrak{E}\right), \\
& \mathfrak{A}_{3}=-\left(\mathfrak{E}^{T} \mathfrak{C}^{-1} \mathfrak{D}\right)\left(\mathfrak{D}^{T} \mathfrak{C}^{-1} \mathfrak{D}\right)^{-1}\left(\mathfrak{D}^{T} \mathfrak{C}^{-1} \mathfrak{E}\right)+\left(\mathfrak{E}^{T} \mathfrak{C}^{-1} \mathfrak{E}\right) .
\end{aligned}
$$

$\mathfrak{F}_{1}$ and $\mathfrak{F}_{2}$ can be derived as

$$
\begin{aligned}
& \mathfrak{F}_{1}=\left(\begin{array}{ccc}
\mathbf{B} & \mathbf{0} & \mathbf{0}
\end{array}\right)\left(\mathfrak{D}^{T} \mathfrak{C}^{-1} \mathfrak{D}\right)^{-1} \\
& \mathfrak{F}_{2}=-\left(\mathfrak{E}^{T} \mathfrak{C}^{-1} \mathfrak{D}\right)\left(\mathfrak{D}^{T} \mathfrak{C}^{-1} \mathfrak{D}\right)^{-1}
\end{aligned}
$$

Remark 2.10. From theorem 2.7 it follows that

$$
\boldsymbol{\eta}_{2}^{T} \boldsymbol{A}_{3} \boldsymbol{\eta}_{2}=\left(\mathfrak{D} \boldsymbol{\eta}_{1}+\mathfrak{E} \boldsymbol{\eta}_{2}\right)^{T} \mathfrak{C}^{-1}\left(\mathfrak{D} \boldsymbol{\eta}_{1}+\mathfrak{E} \boldsymbol{\eta}_{2}\right)>0 \text { for all } \boldsymbol{\eta}_{2} \in \mathbb{R}^{3 L} \backslash\{0\}
$$

where

$$
\boldsymbol{\eta}_{1}=-\left(\mathfrak{D}^{T} \mathfrak{C}^{-1} \mathfrak{D}\right)^{-1} \mathfrak{D}^{T} \mathfrak{C}^{-1} \mathfrak{E} \boldsymbol{\eta}_{2}
$$

Therefore $\mathfrak{A}_{3}$ is a symmetric positive definite matrix.

Remark 2.11. By using the above remark, we can take one more step to solve the system for

$$
\left(\begin{array}{c}
\tilde{\lambda}^{l} \\
\tilde{\boldsymbol{\lambda}}^{+} \\
\tilde{\boldsymbol{\lambda}}^{-}
\end{array}\right)=\mathfrak{A}_{3}^{-1}\left(\mathfrak{A}_{2}^{T} \tilde{\mathbf{u}}-\mathfrak{F}_{2} \frac{\mathrm{d}}{\mathrm{d} t}\left(\begin{array}{c}
\mathbf{0} \\
\mathbf{F}_{2}^{+} \\
\mathbf{F}_{2}^{-}
\end{array}\right)\right)
$$

whence the system $(2.58)$ is reduced to

$$
\left(\mathfrak{A}_{1}+\mathfrak{A}_{2} \mathfrak{A}_{3}^{-1} \boldsymbol{A}_{2}^{T}\right) \frac{\mathrm{d}}{\mathrm{d} t} \tilde{\mathbf{u}}+\mathbf{A} \tilde{\mathbf{u}}=\left(\mathfrak{F}_{1}+\boldsymbol{A}_{2} \mathfrak{A}_{3}^{-1} \mathfrak{F}_{2}\right) \frac{\mathrm{d}}{\mathrm{d} t}\left(\begin{array}{c}
\mathbf{0} \\
\mathbf{F}_{2}^{+} \\
\mathbf{F}_{2}^{-}
\end{array}\right)
$$

Remark 2.12. Note that in computation of $\mathfrak{A}_{1}, \mathfrak{A}_{2}$ and $\mathfrak{A}_{3}$, the inverse of the matrix $\mathfrak{D} \mathfrak{C}^{-1} \mathfrak{D}$ is needed. In Remark 2.9 we have seen that this inverse can be calculated per finite element. This is an important fact in using the hybridization technique. 
Letting $\Delta t$ be the time step and $\left(\tilde{\mathbf{u}}^{n}, \tilde{\boldsymbol{\lambda}}^{l, n}, \tilde{\boldsymbol{\lambda}}^{+, n}, \tilde{\boldsymbol{\lambda}}^{-, n}\right)$ the approximation of the solution vector $\left(\tilde{\mathbf{u}}, \tilde{\boldsymbol{\lambda}}^{l}, \tilde{\boldsymbol{\lambda}}^{+}, \tilde{\boldsymbol{\lambda}}^{-}\right)$ at $t=t_{n}=n \Delta t$. Then the nonlinear system of equation (2.58) resulting from backward Euler is:

$$
\begin{aligned}
& \left(\begin{array}{c}
\mathbf{A} \tilde{\mathbf{u}}^{n+1} \\
\mathbf{0}
\end{array}\right)+\left(\begin{array}{cc}
\mathfrak{A}_{1}\left(\varphi_{h}^{n+1}, c_{h}^{+, n+1}, c_{h}^{-, n+1}\right) & \mathfrak{A}_{2}\left(\varphi_{h}^{n+1}, c_{h}^{+, n+1}, c_{h}^{-, n+1}\right) \\
\mathfrak{A}_{2}^{T}\left(\varphi_{h}^{n+1}, c_{h}^{+, n+1}, c_{h}^{-, n+1}\right) & -\mathfrak{A}_{3}\left(\varphi_{h}^{n+1}, c_{h}^{+, n+1}, c_{h}^{-, n+1}\right)
\end{array}\right) \\
& \times\left(\begin{array}{c}
\frac{\tilde{\mathbf{u}}^{n+1}-\tilde{\mathbf{u}}^{n}}{\hat{A}_{t}^{t}} \\
\tilde{\boldsymbol{\lambda}}^{, n+1} \\
\tilde{\boldsymbol{\lambda}}^{+, n+1} \\
\tilde{\boldsymbol{\lambda}}^{-, n+1}
\end{array}\right)=\left(\begin{array}{c}
\mathbf{F}_{1} \\
\mathbf{0}
\end{array}\right)+\left(\begin{array}{l}
\mathfrak{F}_{1}\left(\varphi_{h}^{n+1}, c_{h}^{+, n+1}, c_{h}^{-, n+1}\right) \\
\mathfrak{F}_{2}\left(\varphi_{h}^{n+1}, c_{h}^{+, n+1}, c_{h}^{-, n+1}\right)
\end{array}\right)
\end{aligned}
$$

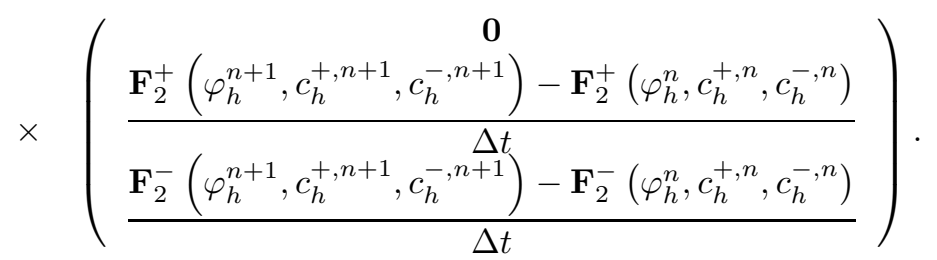

The assumption that $c_{h}^{\beta}, \beta=+,-$, and $\varphi_{h}$ are known transforms the system into a linear one. For $\varphi_{h}$ the value at the old time step is taken. Thus the value of $c_{h}^{f c}$ at the old time level is known and therefore the difference $c_{h}^{+}-c_{h}^{-}$by electroneutrality. Define $c^{ \pm}=c^{+}+c^{-}$, then the solution $\mu_{h}^{\beta}$ of the linear system (2.67) and (2.57) for fixed $c_{h}^{\beta}, \beta=+,-$ can be denoted symbolically by $\left(\mu_{h}^{+}, \mu_{h}^{-}\right)=\mathrm{A}^{-1}\left(c_{h}^{ \pm}\right) \mathbf{b}\left(c_{h}^{ \pm}\right)$, where $\mathbf{A}$ and $\mathbf{b}$ represent the matrix and right-hand side of the linear system. Now $c_{h}^{ \pm}$can be computed by the third relation in the secondary equations in (1.1), i.e. $c_{h}^{ \pm}=\mathrm{f}\left(\mu_{h}^{+}, \mu_{h}^{-}, c_{h}^{ \pm}\right)$, where the dependence of $\mathrm{f}$ on $c_{h}^{ \pm}$is generated by the activity coefficient $f^{\beta}$. Ergo, we obtain the non-linear system

$$
c_{h}^{ \pm}=\mathrm{f}\left(\mathrm{A}^{-1}\left(c_{h}^{ \pm}\right) \mathrm{b}\left(c_{h}^{ \pm}\right), c_{h}^{ \pm}\right) .
$$

This system can be solved by an iterative procedure for non-linear system (see Algorithm 1).

In each iteration a linear system has to be solved. It should be observed that different scales are apparent in (2.58) that will result in a poorly scaled matrix in the linear system. Therefore the matrix $A$ is replaced by the scaled matrix DAD, where the diagonal matrix $\mathrm{D}$ is defined by

$$
\mathrm{D}^{2}=\operatorname{diag}\left(\frac{L}{\Delta t\left(2 \mu_{s}+\lambda_{s}\right)}, \frac{L}{K}, \frac{R T L}{D^{+} \varphi_{0} c_{0}^{f c}}, \frac{R T L}{D^{-} \varphi_{0} c_{0}^{f c}}\right) .
$$

Here $L$ is a representative length scale.

\section{Numerical Simulations}

\subsection{The system of equations}

In this section the element contributions to the matrices and right-hand sides in (2.58) is computed.

The element contribution to the elasticity matrix $\mathbf{A}$ and $\mathbf{B}$ in (2.58) can be calculated in an standard way. To calculate the element contribution to the matrices $\mathbf{C}^{\beta \gamma}, \beta, \gamma=l,+,-$, we need to consider the following analysis. After linearizing the problem it is enough to calculate the following matrix which we call it $\mathbf{C}$. Considering (2.30) we have

$$
\left(\mathbf{C}_{J^{(T)} \times J^{(T)}}^{(T)}\right)_{i j}=\int_{T} \mathbf{v}_{i}^{(T)} \cdot \mathbf{v}_{j}^{(T)} \mathrm{d} \mathbf{x}=\int_{\hat{T}} \hat{\mathbf{C}}^{(T)} \hat{\mathbf{v}}_{i} \cdot \hat{\mathbf{v}}_{j} \mathrm{~d} \hat{\mathbf{x}},
$$




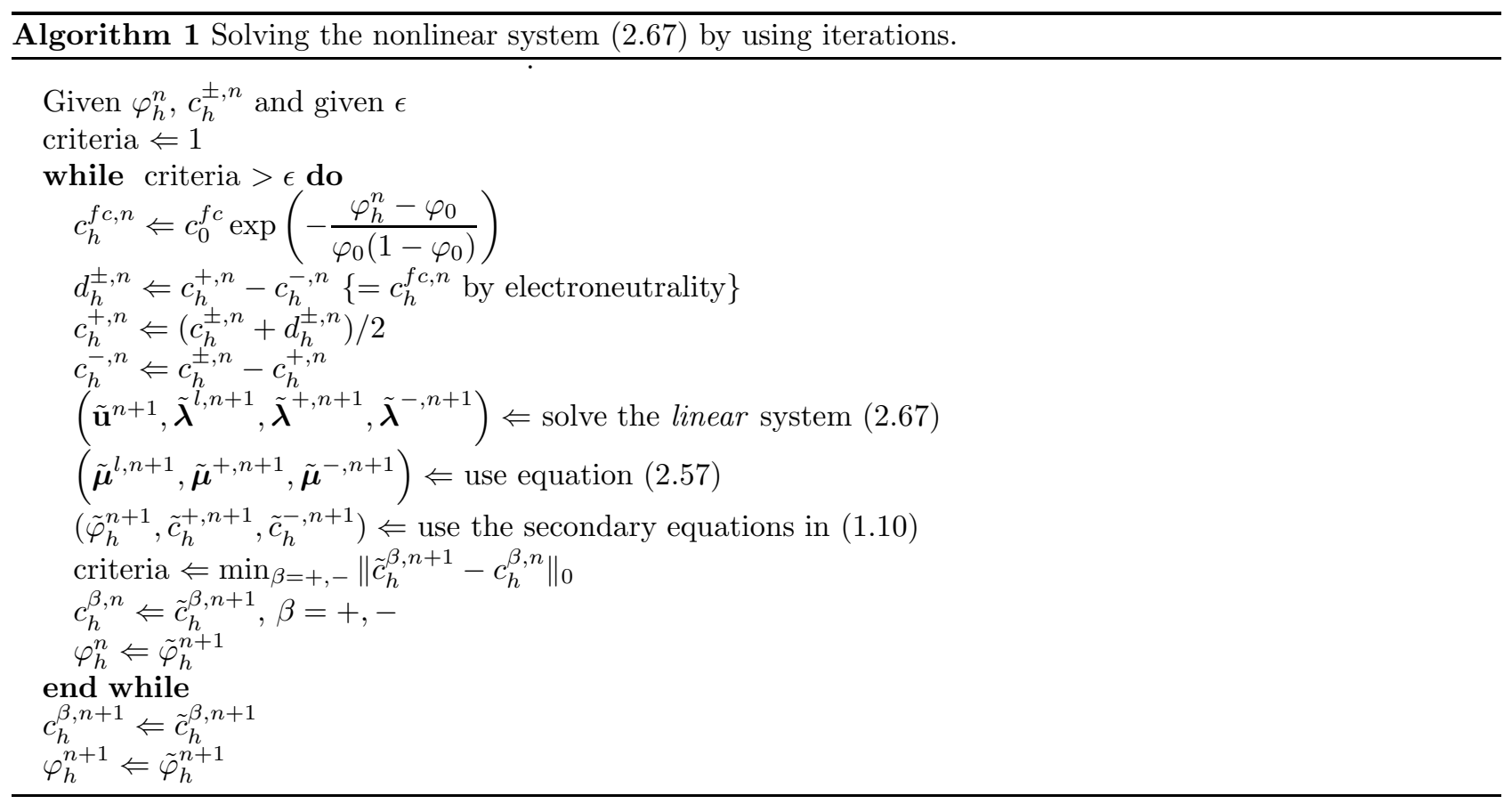

where $\hat{\mathbf{C}}^{(T)}(\hat{x})=\left(\operatorname{det} \mathbf{B}_{n}\right)^{-1} \mathbf{B}_{n}^{T} \mathbf{B}_{n}$ and $\hat{\mathbf{v}}_{i}(\hat{\mathbf{x}})=\left(\operatorname{det} \mathbf{B}_{n}\right) \mathbf{B}_{n}^{-1} \mathbf{v}_{i}^{(T)}(\mathbf{x}), \mathbf{x} \in T$, is considered. Note that the local basis functions $\hat{\mathbf{v}}_{i}, i=1, \ldots, J^{(T)}$, only depend on the reference element $\hat{T}$. If the notations $\hat{\mathbf{v}}_{i}(\hat{\mathbf{x}})=$ $\left[\hat{v}_{i k}(\hat{\mathbf{x}})\right]_{k=1, \ldots, n}$ and $\hat{\mathbf{C}}^{(T)}=\left[\hat{c}_{k l}^{(T)}\right]_{k, l=1, \ldots, n}$ are used, then

$$
\int_{\hat{T}} \hat{\mathbf{C}}^{(T)} \hat{\mathbf{v}}_{i} \cdot \hat{\mathbf{v}}_{j} \mathrm{~d} \hat{\mathbf{x}}=\sum_{k=1}^{n} \sum_{l=1}^{n} \hat{c}_{k l}^{(T)}\left(\mathbf{A}_{k l}\right)_{i j}
$$

where the matrices

$$
\left(\left(\mathbf{A}_{k l}\right)_{J^{(T)} \times J^{(T)}}\right)_{i j}=\int_{\hat{T}} \hat{v}_{i k} \hat{v}_{j l} \mathrm{~d} \hat{\mathbf{x}}
$$

only depend on the reference element $\hat{T}$. Note that $\hat{c}_{k l}^{(T)}=\hat{c}_{l k}^{(T)}$ and $\mathbf{A}_{k l}=\mathbf{A}_{l k}$, thus

$$
\left.\left(\mathbf{C}_{J^{(T)} \times J^{(T)}}^{(T)}\right)\right)_{i j}=\sum_{k=1}^{n} \hat{c}_{k k}^{(T)}\left(\mathbf{A}_{k k}\right)_{i j}+\sum_{k=1}^{n} \sum_{l=1}^{n-1} \hat{c}_{k l}^{(T)}\left(\mathbf{A}_{k l}+\mathbf{A}_{l k}\right)_{i j},
$$

where all matrices in the summations are symmetric.

In [9], an overview of the matrices $\mathbf{A}_{k k}$ and $\mathbf{A}_{k l}+\mathbf{A}_{l k}, k \neq l$, corresponding to various choice of the reference element $\hat{T}$ is given. 
Using Green's formula, (2.34) and (2.29), simple formulas can be derived for the element contributions of the remaining matrices and right hand sides in (2.58), namely

$$
\begin{aligned}
\left(D_{J^{(T)} \times 1}^{(T)}\right)_{i} & =-\int_{T} \nabla \cdot \mathbf{v}_{i}^{(T)} \mathrm{d} \mathbf{x}=-1, \\
\left(G_{J^{(T)} \times J^{(T)}}^{(T)}\right)_{i k} & =\int_{e_{k}^{(T)}} \mathbf{n}_{T} \cdot \mathbf{v}_{i}^{(T)} \mathrm{d} s=\delta_{i k}, \\
F_{1}^{\beta(T)} & =\left\{\begin{array}{ll}
-\tilde{\mu}_{i n}, & \text { if } e_{i} \subset \overline{\Gamma_{p}^{D}} \\
0 & \text { otherwise. }
\end{array}, \beta=l,+,-.\right.
\end{aligned}
$$

The computation of $\left(\mathcal{D}_{1}^{T} \mathcal{C}^{-1} \mathcal{D}_{1}\right)^{-1}$ is essential when determining the matrices $\mathfrak{A}_{i},(i=1,2,3)$ in $(2.59)-(2.61)$. This matrix is a block diagonal matrix (Rem. 2.9) and it can thus be computed at the finite element level. The matrices $\mathfrak{A}_{i}$ are obtained by assembling its element contributions. The right hand side vectors $\mathfrak{F}_{1}$ and $\mathfrak{F}_{2}$ can be computed in an analogous way.

\subsection{Confined consolidation and free swelling experiments}

In this section, numerical solutions are calculated for confined consolidation and free swelling. To verify the method, solutions are compared with the analytical solutions from [10]. The homogeneous sample is placed frictionless in a holder. The opposite figure illustrates the experimental setup. At the bottom $X=0,(X$ is the Lagrangian coordinate) the sample is in contact with a glass filter saturated by a sodium chloride solution. An impermeable piston is placed on the top of the sample, $X=L$, where an external mechanical load is applied. The sample was made out of a hydrogel. A bathing solution flowed through a porous glass filter at the bottom of the sample.

\section{Consolidation}



In the consolidation experiment, we apply a load on the piston to be equal to $f(t)=-f_{0} \mathcal{H}\left(t-t_{0}\right)$, with $\mathcal{H}\left(t-t_{0}\right)$ the Heaviside function and $f_{0}>0$. For the initial values of electro-chemical potentials we choose

$$
\mu_{0}^{l}=2 R T c_{\text {out }}, \quad \mu_{0}^{\beta}=-R T \ln \frac{c_{\text {out }}}{c} .
$$

By Donnan equilibrium, we know that the electro-chemical potentials are continuous at the boundary, i.e., $\mu_{i n}^{\beta}=\mu_{o u t}^{\beta}, \beta=l,+,-$, where subscript "in" and "out" stand for inner and outer solution, respectively. Assume $\Gamma_{\text {in }}^{+}=\Gamma_{\text {in }}^{-}=\Gamma, \Gamma_{\text {out }}^{+}=\Gamma_{\text {out }}^{-}=1$ and $p_{\text {out }}=0$ and $\xi_{\text {out }}=0$, then

$$
\begin{aligned}
& \mu_{\text {in }}^{l}=\mu_{\text {out }}^{l}=\mu_{0}^{l}+p_{\text {out }}-2 R T \Gamma_{\text {out }} c_{\text {out }}=0, \\
& \mu_{\text {in }}^{\beta}=\mu_{\text {out }}^{\beta}=\mu_{\text {out }}^{\beta}+z^{\beta} F \xi_{\text {out }}+R T \Gamma_{\text {out }} \ln \frac{c_{\text {out }}}{c}=0, \quad \beta=+,-.
\end{aligned}
$$

Therefore, the initial condition for the displacement and electro-chemical potentials with respect to the steady state initial state $t=t_{0}$ are:

$$
\begin{aligned}
{\left[\begin{array}{lll}
\mu^{l} & \mu^{+} & \mu^{-}
\end{array}\right](0, t) } & =\left[\begin{array}{lll}
0 & 0 & 0
\end{array}\right] \\
{\left[\begin{array}{lll}
\mu^{l} & \mu^{+} & \mu^{-}
\end{array}\right]\left(X, t_{0}\right) } & =\left[\begin{array}{lll}
\mu_{t_{0}}^{l} & \mu_{t_{0}}^{+} & \mu_{t_{0}}^{-}
\end{array}\right], \\
u(0, t) & =0 \\
u\left(X, t_{0}\right) & =0
\end{aligned}
$$


where $\left[\begin{array}{ccc}\mu_{t_{0}}^{l} & \mu_{t_{0}}^{+} & \mu_{t_{0}}^{-}\end{array}\right]=\left[\begin{array}{lll}f_{0} & 0 & 0\end{array}\right]$.

Free swelling

In free swelling experiment, we change the concentration of the external salt solution. For the initial values of electro-chemical potentials we choose

$$
\mu_{0}^{l}=2 R T c_{\text {out }}, \quad \mu_{0}^{\beta}=-R T \ln \frac{c_{\text {out }}}{c} .
$$

The initial and boundary conditions for the displacement and electro-chemical potentials with respect to the steady state initial state $t=t_{0}$ are:

$$
\begin{aligned}
{\left[\begin{array}{lll}
\mu^{l} & \mu^{+} & \mu^{-}
\end{array}\right](0, t) } & =\left[\begin{array}{lll}
\mu_{i n}^{l} & \mu_{i n}^{+} & \mu_{i n}^{-}
\end{array}\right] \\
{\left[\begin{array}{lll}
\mu^{l} & \mu^{+} & \mu^{-}
\end{array}\right]\left(X, t_{0}\right) } & =\left[\begin{array}{lll}
\mu_{t_{0}}^{l} & \mu_{t_{0}}^{+} & \mu_{t_{0}}^{-}
\end{array}\right] \\
u(0, t) & =0 \\
u\left(X, t_{0}\right) & =0
\end{aligned}
$$

in which $\mu_{t_{0}}^{\beta}=0, \beta=l,+,-$, and

$$
\left\{\begin{array}{l}
\mu_{\text {in }}^{l}=-2 R T \Delta c_{\text {out }}, \\
\mu_{\text {in }}^{+}=R T \ln \frac{c_{\text {out }}\left(t_{0}^{+}\right)}{c_{\text {out }}\left(t_{0}^{-}\right)}, \\
\mu_{\text {in }}^{-}=R T \ln \frac{c_{\text {out }}\left(t_{0}^{+}\right)}{c_{\text {out }}\left(t_{0}^{-}\right)}
\end{array}\right.
$$

$\Delta c_{\text {out }}$ is the change in the external concentration and $t_{0}^{+}$and $t_{0}^{-}$are the time just after and before $t_{0}$ when chemical loading is applied.

\subsection{Results}

In this section, the results for the confined consolidation and the free swelling are prescribed. For both computations, the parameters in the table are taken. These two experiments are taken from [7] and our aim is to validate the numerical solutions by analytical solutions described in [10].

As mentioned in the previous section, an uniaxial confined swelling and compression experiment is performed on a cylindrical sample of cartilage substitute. This sample, with the diameter of $4 \mathrm{~mm}$ and the height of approximately $1 \mathrm{~mm}$ is put in an insulating conforming ring. A piston on the top of the sample is loaded mechanically. A bathing solution flows through a porous glass filter at the bottom of the sample. A change of the salt concentration of this solution generates a change in the boundary of ion concentrations and electro-chemical potentials as well

\begin{tabular}{|ccc|}
\hline Parameter & Unit & Value \\
\hline $2 \mu_{s}+\lambda_{s}$ & $\mathrm{MPa}$ & $4 \times 10^{3}$ \\
$K$ & $\mathrm{~m}^{4} \cdot \mathrm{N}^{-1} \cdot \mathrm{s}^{-1}$ & $1.0 \times 10^{-18}$ \\
$c^{f c}$ & $\mathrm{~mol} \cdot \mathrm{m}^{-3}$ & $-2 \times 10^{2}$ \\
$c_{\text {out }}$ & $\mathrm{mol} \cdot \mathrm{m}^{-3}$ & $1 \times 10^{2}$ \\
$\varphi$ & & 0.1 \\
$D^{+}$ & $\mathrm{m}^{2} \cdot \mathrm{s}^{-1}$ & $13.3 \times 10^{-10}$ \\
$D^{-}$ & $\mathrm{m}^{2} \cdot \mathrm{s}^{-1}$ & $20.3 \times 10^{-10}$ \\
$R$ & $\mathrm{~J} \cdot \mathrm{mol}^{-1} \cdot K^{-1}$ & 8.3145 \\
$T$ & $\mathrm{~K}$ & 293 \\
$F$ & $\mathrm{C} \cdot \mathrm{mol}^{-1}$ & 96484.6 \\
$\Gamma$ & & 0.9 \\
\hline
\end{tabular}
as pressure and voltage.

During the experiment, the mechanical and chemical load are varied. Inspired by this, two numerical simulations are considered.

Note that the problems of confined consolidation and free swelling are one dimensional with variation in the $y$ direction only. Considering the extension of our model to higher dimensions, we would prefer to implement these one-dimensional models in a horizontally-fixed displacement two-dimensional geometry. We use a rectangle in which only vertical displacement (direction of force) is allowed with 32 vertical elements and one horizontal 




(a) Displacement at $y=L$

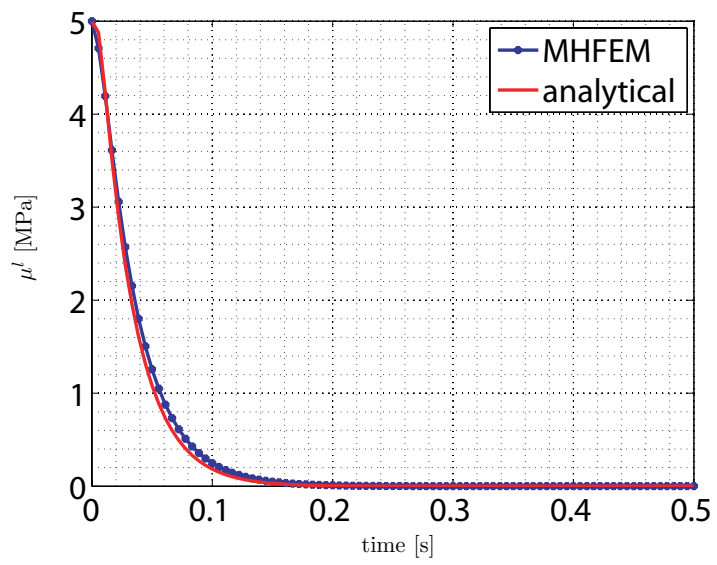

(c) $\mu^{l}$ at $y=L$

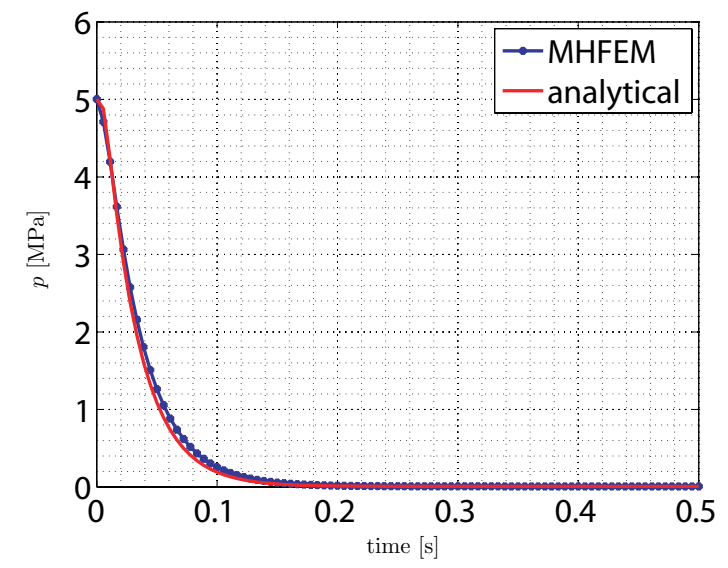

(b) Pore pressure at $y=L$

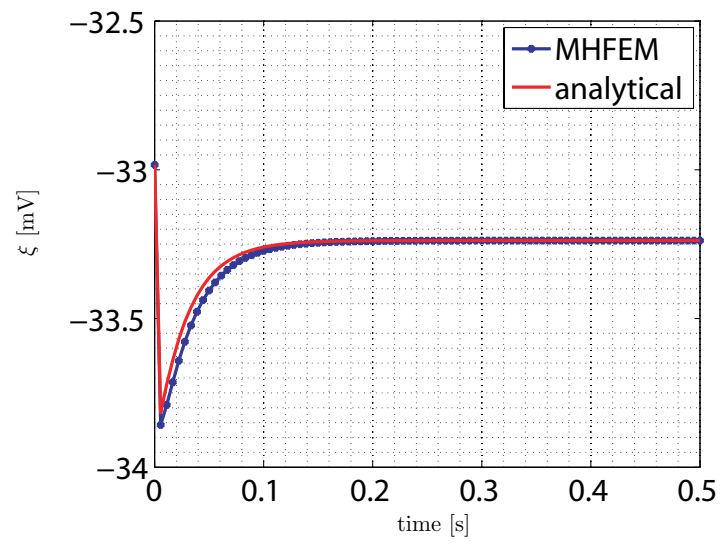

(d) electrical potential at $y=L$

FIGURE 1. Analytical-MHFEM comparison of the solutions for the confined consolidation (I).

element. Successively, 30 increments of 20 seconds, 10 of 50 seconds and 7 of 100 seconds are used for time discretisation.

Analytical solutions for displacement, fluid pressure, electro-chemical potentials and flows for both of problems have been derived in [10]. Representative results from mixed hybrid finite element are compared to these solutions.

For the consolidation experiment, an inward force $f_{0}=5 \mathrm{MPa}$ is applied to the top no-flow boundary, and at the bottom rigid boundary, the porous medium is in contact with an electroneutral bathing solution. All boundary conditions are described in (3.9).

The implicit Euler backward scheme is used for the time discretisation. The nonlinear system (2.66) is solved based on iterations for $c^{ \pm}$. The criteria for convergence of this iteration is $10^{-12}$. The average iterations for this problem is 10 iteration per time step and it is reduced from 15 iterations in the beginning to one when sample reaches equilibrium. We use a direct solver for the linear system. In fact, a direct solver for our one-dimensional problem is a good choice but we are aware of the fact that for higher-dimensional problems an iterative solver should be considered. 


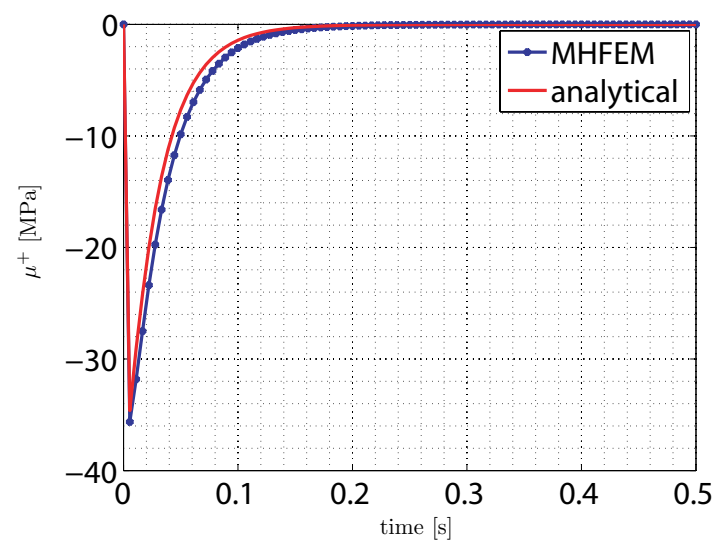

(a) $\mu^{+}$at $y=L$

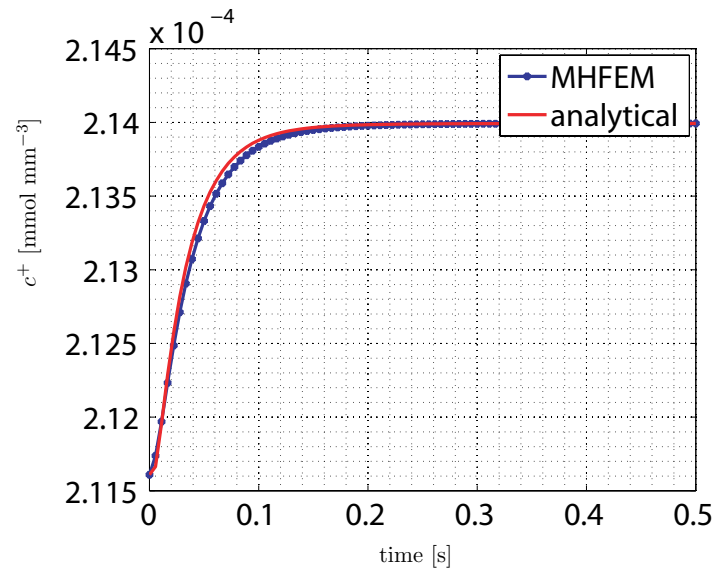

(c) cation concentration at $y=L$

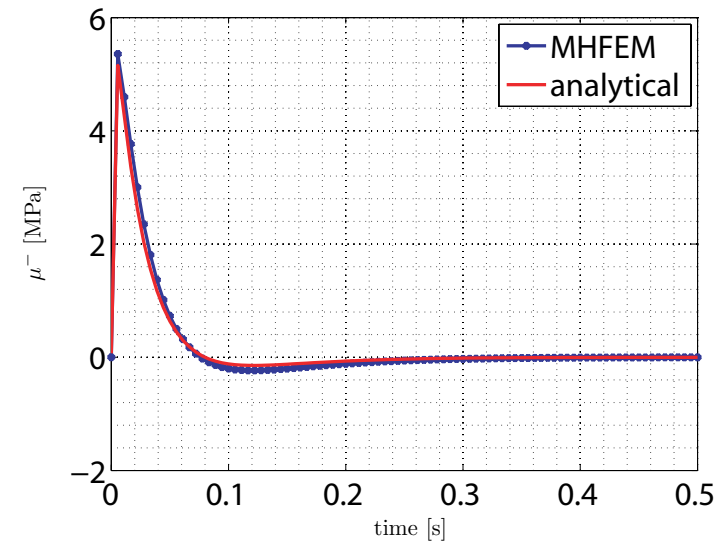

(b) $\mu^{-}$at $y=L$

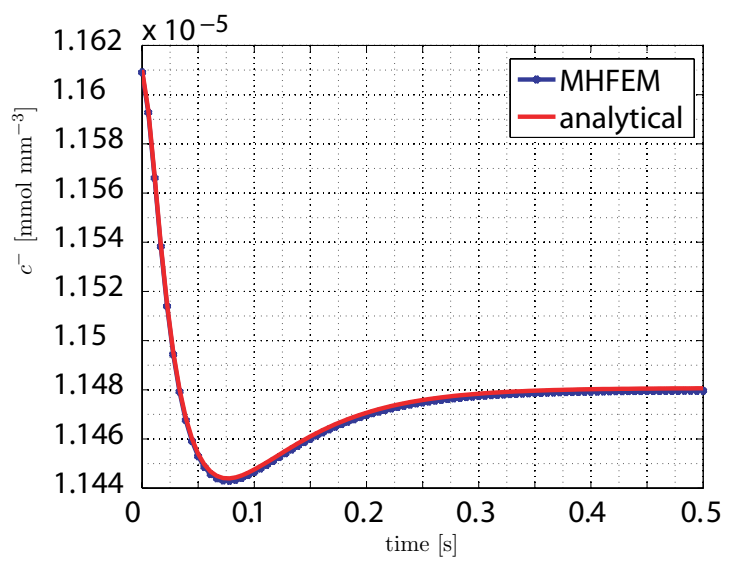

(d) anion concentration at $y=L$

FiguRE 2. Analytical-MHFEM comparison of the solutions for the confined consolidation (II).

Note that in this experiment, all the unknowns change immediately at $t=t_{0} \mathrm{~s}$, thus another equilibrium will be establish at infinite time. At the final equilibrium, the electro-chemical potentials $\mu^{\beta}, \beta=l,+,-$ have the same value as the values in the initial state. However, the stress and fluid pressure have changed, since the porous medium is compressed, Figures 1 and 2 display the comparison between the analytical solution and mixed hybrid finite element solutions for confined consolidation.

For the free swelling experiment, the initial and boundary conditions are chosen from (3.11). In this experiment, we decrease the external salt concentration with a small amount from $c_{\text {out }}=1 \times 10^{2} \mathrm{~mol} \cdot \mathrm{m}^{-3}$ to $c_{\text {out }}=0.995 \times 10^{2} \mathrm{~mol} \cdot \mathrm{m}^{-3}$. The displacement, pore pressure, electrical potential, electro-chemical potentials and ion concentrations are displayed in Figures 3 and 4. The pore pressure increases from the initial value to the maximum value $0.0041 \mathrm{MPa}$ at $0.1648 \mathrm{~h}$

The results obtained, despite the relative coarseness of the meshes are quite good. As it was expected from the mixed method, the fluid flow and ion inflows and outflows are equal per element and per time step. All functions were written in MATLAB and were run on a pentium IV $(2.66 \mathrm{GHz})$.

Due to the nature of the hybridization method, the element matrix computation time and the inverse of the block diagonal matrix to get the global matrix is markedly smaller than the same procedure in the finite element code. Another reason for the hybrid method's better performance is the relatively shorter time required 




(a) Displacement at $y=L$

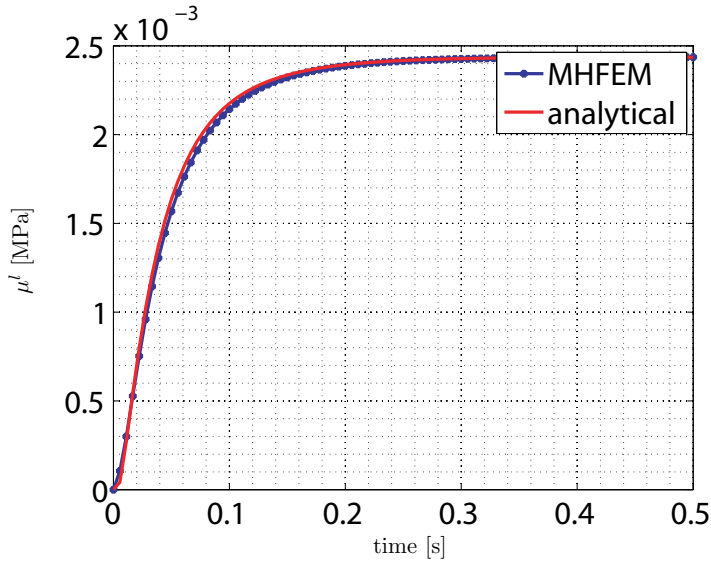

(c) $\mu^{l}$ at $y=L$

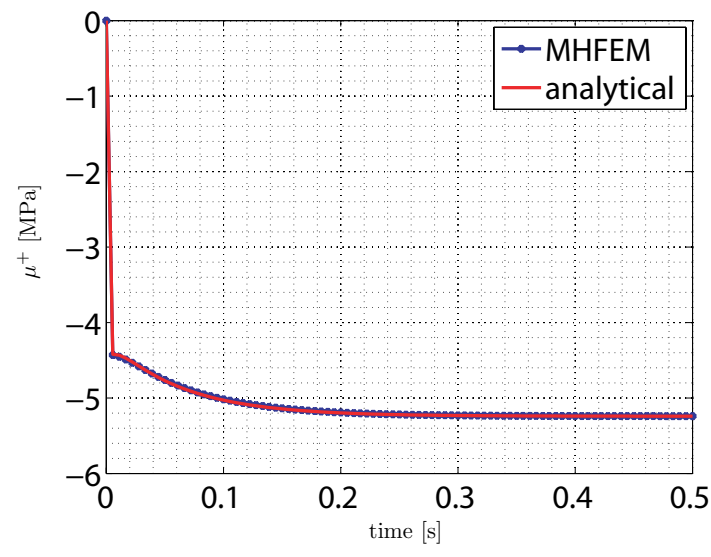

(e) $\mu^{+}$at $y=L$

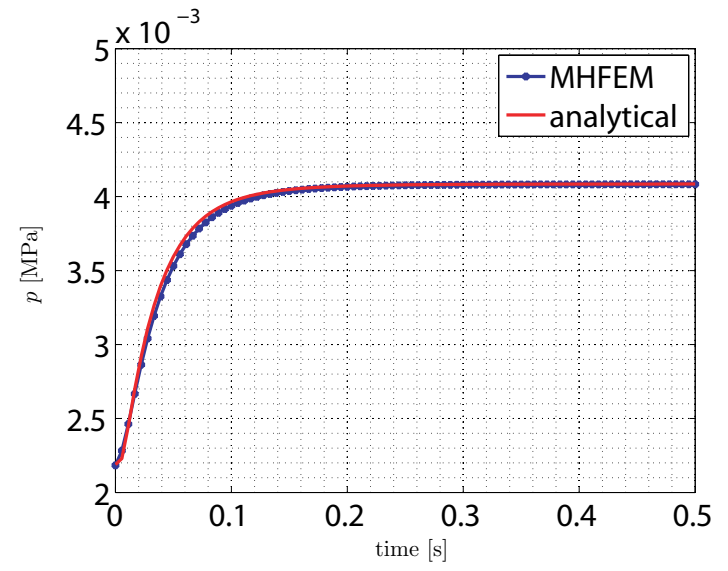

(b) Pore pressure at $y=L$

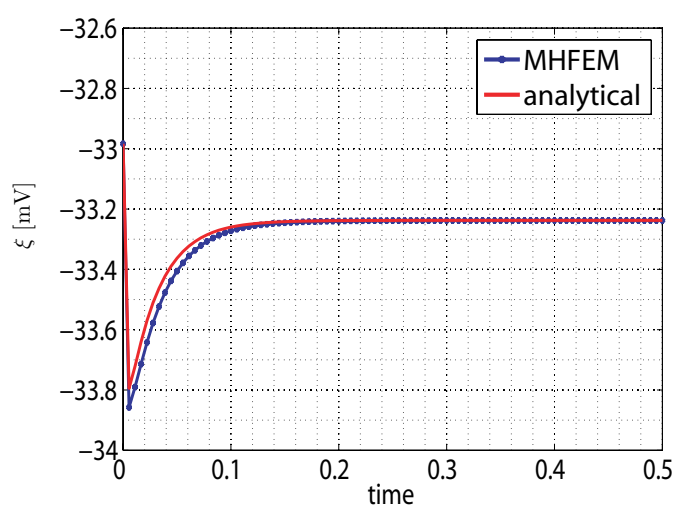

(d) electrical potential at $y=L$

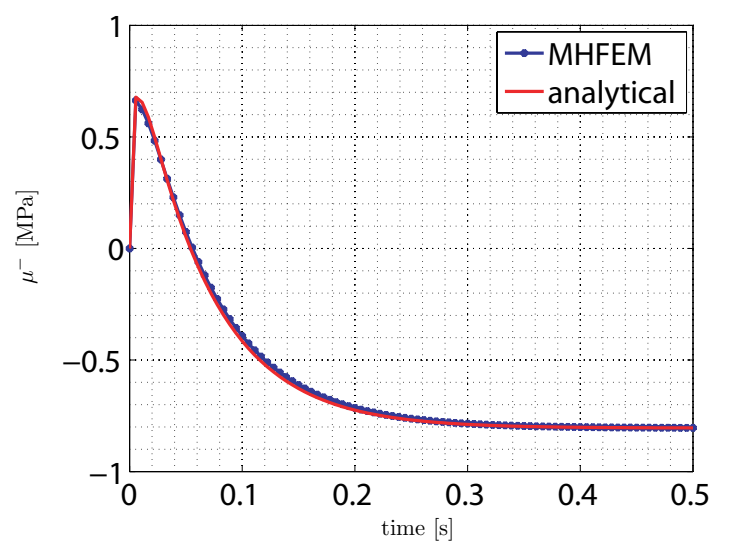

(f) $\mu^{-}$at $y=L$

Figure 3. Analytical-MHFEM comparison of the displacement and pore pressure for confined swelling (I). 


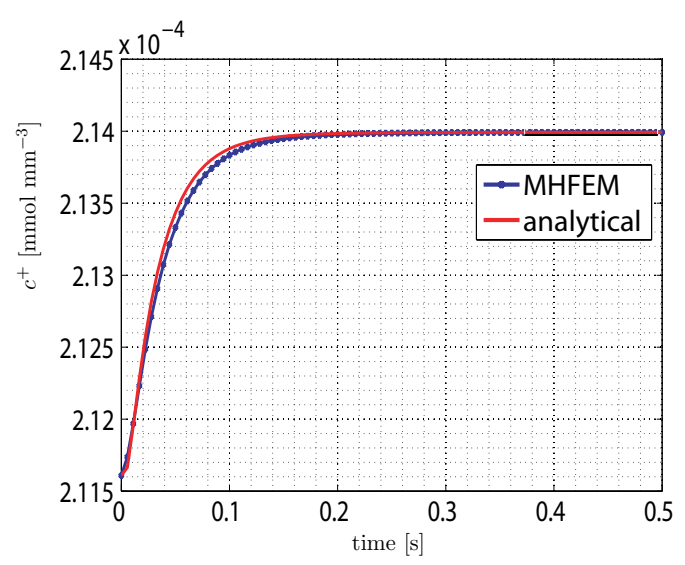

(a) cation concentration at $y=L$



(b) anion concentration at $y=L$

FiguRE 4. Analytical-MHFEM comparison of the displacement and pore pressure for confined swelling (II).

to compute the right-hand side at each iteration and each time step. This is due to the fact that $\left(\mathfrak{D}^{T} \mathfrak{C}^{-1} \mathfrak{D}\right)^{-1}$ in (2.62) and (2.63) is computed per element in forming the stiffness matrix. Thus the force vector is computed from stored element level values $\varphi$ and $c^{\beta}$ at the previous time step independently for each element. In contrast, the right-hand side for the finite element method [17] is computed at the global level, requiring the multiplication of the entire stiffness matrix. Therefore the finite element method needs more CPU time due to the size of the matrices involved.

In conclusion, for the large problems for which a three-dimensional geometry is considered, the hybrid method offers significant advantages in both the amount of storage required and the CPU time to obtain a solution.

\subsection{Two-dimensional free swelling}

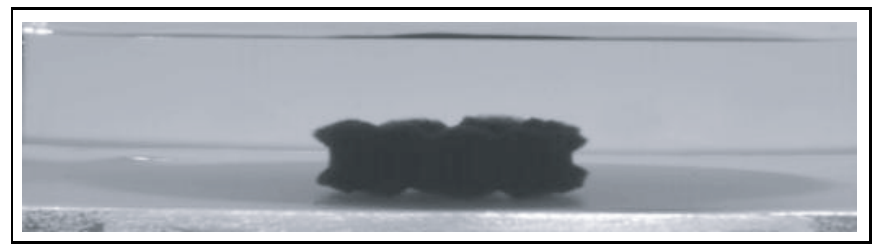

Figure 5. Experiment on hydrogel disc

(J.M. Huyghe, 1999).
In this section, a mixed hybrid finite elements for two-dimensional four-component model is developed.

We consider the swelling cylinder to test the twodimensional hybridization method. We choose an axisymmetric geometry as described below.

A cylinder of height $0.5 \mathrm{~mm}$ and radius $1 \mathrm{~mm}$ is in equilibrium with an external salt solution. The external concentration is reduced from $4.6 \times$ $10^{2} \mathrm{~mol} \cdot \mathrm{m}^{-3}$ to $4 \times 10^{2} \mathrm{~mol} \cdot \mathrm{m}^{-3}$. A change of the salt concentration of this solution generates a change at the boundary of ion concentrations and electro-chemical potentials as well as pressure and voltage.

For this computation we take $\mu_{s}=0.4688 \mathrm{MPa}, \lambda_{s}=0.3125 \mathrm{MPa}, \varphi^{f}=0.7$ and the other parameters are unchanged.

The intersection of the cylinder through the center is made in order to illustrate the swelling. For the boundary conditions of this rectangular shape intersection, we consider no horizontal displacement at the bottom left corner and no vertical displacement in the bottom. The bottom and top plane are considered impermeable. 


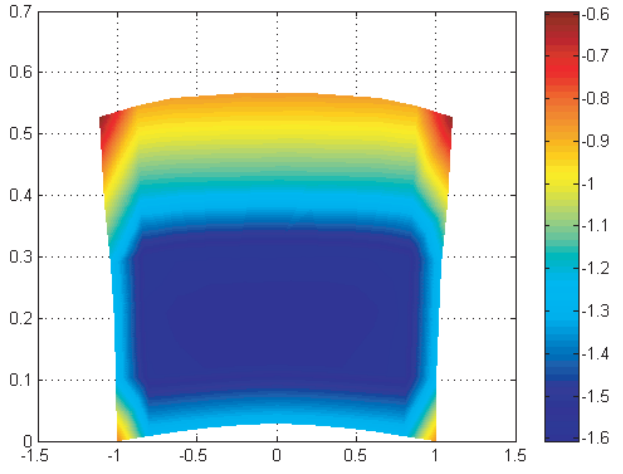

(a) $t=360 \mathrm{~s}$

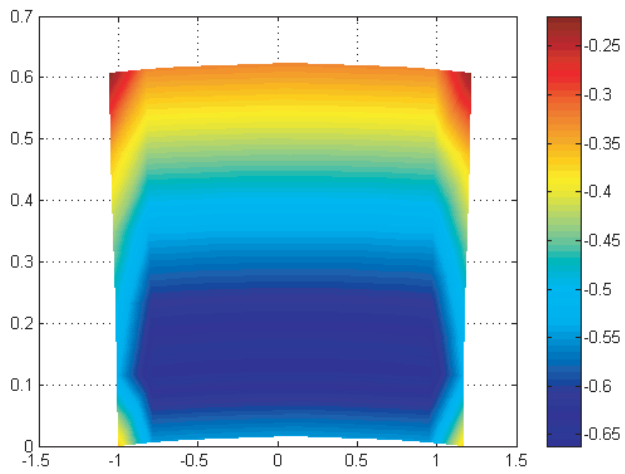

(c) $t=7200 \mathrm{~s}$

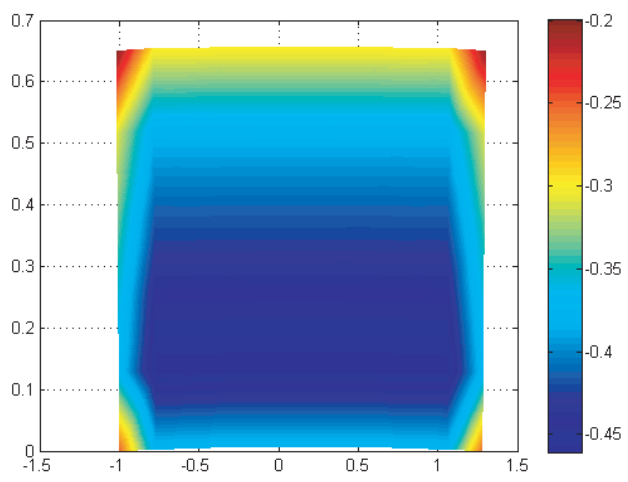

(e) $t=18000 \mathrm{~s}$

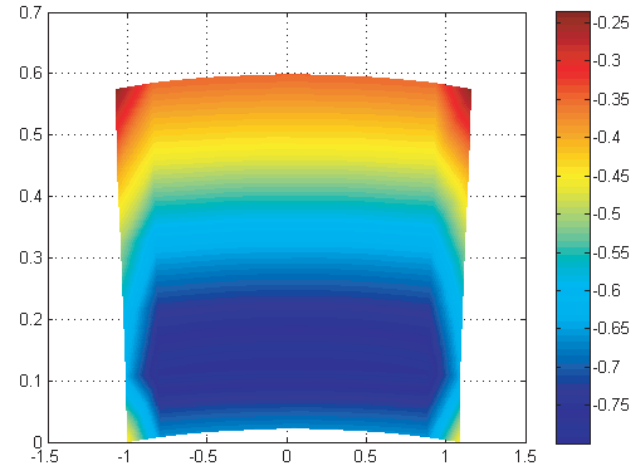

(b) $t=3600 \mathrm{~s}$

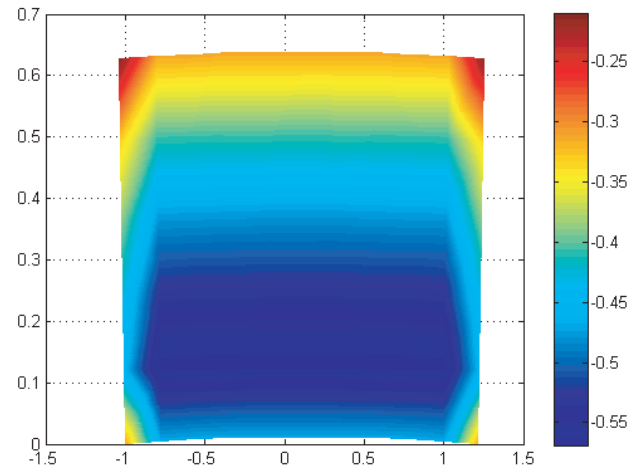

(d) $t=10800 \mathrm{~s}$

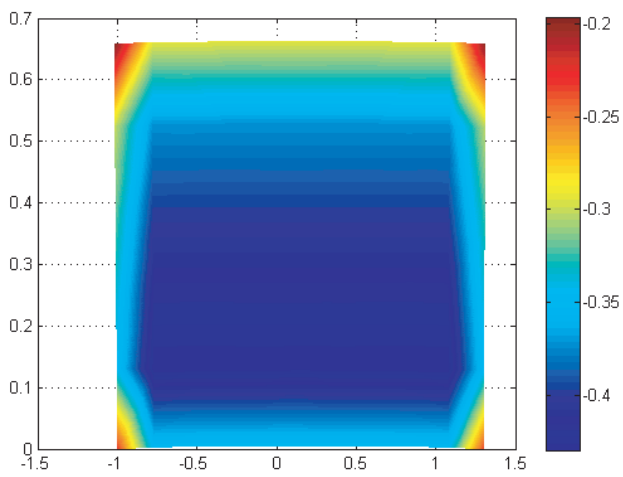

(f) $t=21600 \mathrm{~s}$

Figure 6. Fluid pressure at intersection of cylinder for several times. 
While the external salt concentration is reduced, the cylinder starts to shrink at the top surface where the salt concentration and the medium are in contact. This is due to the fact that the ions need less time to diffuse than the liquid, therefore an ions flow takes the liquid out of the sample and shrinkage happens. Further, the computation shows that after 6 hours the cylinder has regained its initial shape, however it is enlarged.

As it was expected for the higher dimensional geometry the number of iterations for the nonlinear solver is larger in comparison with the one-dimensional case. But thanks to the hybridization technique for less amount of storage it requires. Note that the nonlinear solver takes more iterations for the period ions are diffusing (the shrinkage period), later on while the hydrogel returns to its initial shape, the number of iterations is reduced to one iteration near final time.

Figure $6 \mathrm{a}-\mathrm{f}$ displays the swelling at different times.

\section{Conclusions}

The four-component model in the Lagrangian formulation by assuming infinitesimal deformation for the solid phase is derived [11]. Note that for a finite deformation model it is important that the Lagrangian formulation to be considered. But in this article due to the fact that confined compression and swelling are considered as test problems, infinitesimal assumption is given. This results in a coupled system of nonlinear parabolic differential equation. For the numerical experiments it is clear that an accurate approximation of the flows can be determined by the mixed finite element method. The benefit of the mixed method are apparent for problems with rough tensors of hydraulic conductivity (for example confined compression and swelling method) and especially if the domain is subdivided into very flat sub-domains.

Of course, if one is interested in an accurate approximation of the potential, then the conforming finite element is preferable with a non-uniform time discretisation with a larger steps near the equilibrium. The number of required iterations to solve the nonlinear system is reduced for one-dimensional experiments.

Using the hybridization technique, the mixed finite element method still results in an indefinite system but will less number of degrees of freedom. We use iteration for solving the nonlinear system derived after taking the Backward Euler scheme for the time integration. This algorithm has been tested for one-dimensional confined consolidation and free swelling experiment and the results has been verified with analytical solutions.

A two-dimensional swelling and opening crack problem is tested by using hybridization method. Note that for higher dimensional problem we are aware of using an iterative solver to solve the system of equations. Even more a right preconditioner is needed for our indefinite system. The implicit time discretisation is unconditionally stable, but still we should be careful in choosing the time step to avoid possible oscillations.

\section{REFERENCES}

[1] S.C. Brenner and L.R. Scott, The Mathematical Theory of Finite Element Methods. Springer-Verlag, Berlin-HeidelbergNew York (2002).

[2] F. Brezzi and M. Fortin, Mixed and Hybrid Finite Element Methods. Springer-Verlag, Berlin-Heidelberg-New York (1991).

[3] P.G. Ciarlet, The Finite Element Method for Elliptic Problems, Studies in Mathematics and Its Applications 4. North Holland, Amsterdam (1978).

[4] S. Flügge, Handbuch der physik, Elastizität und plastizität. Springer-Verlag (1958).

[5] B.X. Fraeijs de Veubeke, Displacement and equilibrium models in the finite element method, in Stress Analysis, O.C. Zienkiewicz and G. Holister Eds., John Wiley, New York (1965).

[6] B.X. Fraeijs de Veubeke, An analysis of the convergence of mixed finite element methods. RAIRO Anal. Numér. 11 (1977) $341-354$.

[7] A.J.H. Frijns, A four-component mixture theory applied to cartilaginous tissues. Ph.D. thesis, Eindhoven University of Technology (2001).

[8] J.M. Huyghe and J.D. Janssen, Quadriphasic mechanics of swelling incompressibleporous media. Int. J. Engng. Sci. 35 (1997) 793-802.

[9] E.F. Kaasschieter and A.J.M. Huijben, Mixed-hybrid finite elements and streamline computation for the potential flow problem. Numer. Methods Partial Differ. Equat. 8 (1992) 221-266.

[10] K. Malakpoor, E.F. Kaasschieter and J.M. Huyghe, An analytical solution of incompressible charged porous media. Z. Angew. Math. Mech. 86 (2006) 667-681. 
[11] K. Malakpoor, E.F. Kaasschieter and J.M. Huyghe, Mathematical modelling and numerical solution of swelling of cartilaginous tissues. Part I: Modeling of incompressible charged porous media. ESAIM: M2AN 41 (2007) 661-678.

[12] J.C. Nédélec, Mixed finite elements in $\mathbb{R}^{3}$. Numer. Math. 35 (1980) 315.

[13] J.C. Nédélec, A new family of mixed finite elements in $\mathbb{R}^{3}$. Numer. Math. 50 (1980) 57.

[14] P.A. Raviart and J.M. Thomas, A mixed finite element method for 2nd-order elliptic problems, in Mathematical Aspects of Finite Element Methods, Lecture Note in Mathematics 606, I. Galligani and E. Magenes Eds., Springer, Berlin (1997) $292-315$.

[15] J.E. Roberts and J.M. Thomas, Mixed and hybrid finite element methods, in Handbook of Numerical Analysis, Volume II: Finite Element Methods, P.G. Ciarlet and J.L. Lions Eds., North Holland, Amsterdam (1991) 523-639.

[16] J.M. Thomas, Sur l'analyse numérique des méthodes d'éléments finis hybrides et mixtes. Ph.D. thesis, University Pierre et Marie Curie, Paris (1977).

[17] R. van Loon, J.M. Huyghe, M.W. Wijlaars and F.P.T. Baaijens, 3D FE implementation of an incompressible quadriphasic mixture model. Inter. J. Numer. Meth. Eng. 57 (2003) 1243-1258. 